\title{
Quantitative Untersuchung der expressiven Lexik im Finnischen und seinen nächstverwandten Sprachen
}

\begin{abstract}
Expressive (= descriptive-onomatopoeic) vocabulary forms a coexisting phonological and phonotactic system of its own. It includes - as the material presented in the article shows - many phonemes and phoneme combinations that are rare or even totally unknown in normal vocabulary. In addition, it seems that not all regular phonetic changes have taken place in expressive words. Thus, exceptions to normal language have become characteristic of expressiveness.

The Russian language has had a strong influence on the phonology of the expressive words in all eastern Finnic languages. This is evident in the eastern dialects of Finnish, but can be seen especially clearly in Karelian, Lude and Veps. Expressive vocabulary has been the path through which the phonetic characteristics of Russian have penetrated into the old systems of these languages. In the course of time, foreign affective elements, or at least elements perceived as affective, have displaced several of the original means of representing expressiveness in Lude and Veps.

On the basis of the distribution of the word structures dealt with in the article, it can be concluded that the northern Finnic languages are closely connected also as far as their expressive vocabulary is concerned, particularly Karelian and the eastern dialects of Finnish.
\end{abstract}

\section{Hintergrund}

Die Expressiva, d.h. die deskriptiv-onomatopoetischen Wörter, gelten als verhältnismäßig jung. Allerdings konnte überzeugend dargelegt werden, daß ein großer Teil des finnischen deskriptiv-onomatopoetischen Wortschatzes auf die gemeinsame ostseefinnische Sprachperiode zurückgeht. Die Wurzeln vieler Wörter können natürlich auch erheblich weiter in die Vergangenheit reichen, doch aufgrund der für das expressive Material typischen schwankenden Lautgestalt und unbestimmten Bedeutung läßt sich darüber kein Nachweis führen. Andererseits liegt es 
auf der Hand, daß kontinuierlich neue Wörter entstehen, die vom Typ her der expressiven Lexik zuzuordnen sind. Vgl. Hakulinen 1979: 328, Rytkönen 1940: 184.

Die Anzahl der deskriptiv-onomatopoetischen Wörter kann nur grob geschätzt werden. In allen Sprachformen gibt es jedenfalls zahlreiche expressive Wortstämme. Zudem sind vielfältige lautliche Varianten möglich, und dem jeweiligen Stamm können die unterschiedlichsten Ableitungssuffixe angefügt werden. Auch die Grenzziehung zwischen expressiver und nichtexpressiver Lexik bereitet häufig Schwierigkeiten. Es ist durchaus nicht immer leicht zu entscheiden, ob ein Wort "eindeutig expressiv", "expressiv gefärbt", "zu einem gewissen Grad expressiv geworden" ist oder nur "gelegentlich expressiv verwendet" wird. Einen eigenen Problemkreis stellt die Bestimmung ursprünglich expressiver Wörter dar, die ihren expressiven Charakter bereits verloren haben. Bei der Abgrenzung des deskriptiv-onomatopoetischen Wortschatzes muß man sich demnach - wie beim Verständnis der Expressivität generell auf mehr oder weniger subjektive Kriterien stützen.

Die Verwendung lautmalender Wörter ist sehr wahrscheinlich in der gesprochenen Sprache häufiger als in der Schriftsprache. Ein deutlicher Unterschied besteht sicher auch zwischen dem Dialekt und der sog. gebildeten Umgangssprache: Vermutlich bevorzugt der erstere deskriptiv-onomatopoetische Ausdrücke, während die letztere sie vermeidet (Tuomi 1989: 40-45). Ungeklärt ist vorläufig, in welchem Grad sich die einzelnen ostseefinnischen Sprachen im Gebrauch von Expressiva unterscheiden. Fällt beispielsweise die Tatsache ins Gewicht, daß im Gegensatz zu den anderen osfi. Sprachen das Finnische und das Estnische Kultursprachen mit schriftlicher Tradition sind?

Natürlich haben die deskriptiv-onomatopoetischen Wörter keine einheitlichen Kennzeichen. Dennoch gibt es eine Reihe von Eigenheiten, die für diese Wörter besonders typisch sind. Auf die schwankende Lautform der Expressiva wurde bereits hingewiesen. Für Onomatopoetika ist es sogar charakteristisch, daß ihre Grundstruktur in einem Rahmen oder Kem besteht, der eine allgemeine Bedeutung trägt und durch Konsonanten- oder Vokalwechsel variiert werden kann, wodurch sich unterschiedliche semantische Nuancen ergeben (z. B. hurista surista, rompottaa $\sim$ römpöttää, ratista $\sim$ ritistä). Eine weitere Besonderheit der Expressi- 
va ist die Verwendung von ungewöhnlichen Lauten und Lautverbindungen. Zugespitzt gesagt, bevorzugen die Expressiva vor allem Laute und Lautverbindungen, die im übrigen Wortschatz selten vorkommen. Die Unterschiede sind also in erster Linie quantitativ. Auch Laute und Lautverbindungen, die dem Wortschatz der betreffenden Sprache an sich völlig fremd sind, können bekanntlich in deskriptiv-onomatopoetischen Wörtern auftreten. Quelle der lautlichen Innovationen sind meist Entlehnungen aus Nachbarsprachen oder -dialekten. Fremde Lautelemente scheinen demnach die mit den deskriptiv-onomatopoetischen Wörtern verbundene Affektivität besonders gut zum Ausdruck zu bringen (Itkonen 1966: 133, 204). Selbst von der Regel abweichende Lautveränderungen sind bei Expressiva nicht unbekannt. Ein charakteristisches Beispiel ist die regelwidrige Entwicklung von $s$ und $t$ zur Affrikate $\dot{c}\left(=, t^{\prime} \dot{s}\right) \sim \grave{\zeta}\left(=d^{\prime} \dot{z}\right)$ im Karelischen (Ojansuu 1918: 45-58; s. auch Ravila 1967: 48-51).

Als eine der zentralen Eigenschaften der auf Lautsymbolik fußenden Wörter kann ihre Bedeutungsbreite und -unbestimmtheit gelten: Sie haben keinen genau abgegrenzten semantischen Inhalt, sondern wollen das Benannte mit phonetischen Mitteln schildern. Eine Bedeutungsangabe erweist sich daher oft als schwierig. In Wörterbüchern muß man sich denn auch immer wieder damit behelfen, den Kontext zu nennen, in dem das betreffende Wort verwendet wird, oder expressive Synonyme aufzuzählen. Die deskriptiv-onomatopoetischen Wörter stehen in so enger Verbindung mit den phonetischen Elementen der jeweiligen Sprache und mit ihrer semantischen Interpretation, daß sich durchaus nicht immer exakte Entsprechungen in einer anderen Sprache aufzeigen lassen. Eine ganz andere Situation ergibt sich bei nahe verwandten Sprachen: Die übereinstimmende Lautstruktur und die ähnlichen Ausdrucksformen der Expressivität ermöglichen sogar eine parallele Entwicklung deskriptiv-onomatopoetischer Wörter.

\section{Zu den Verbreitungsrelationen der Expressiva}

Obwohl man bei dem Versuch, das Alter deskriptiv-onomatopoetischer Wörter zu bestimmen, nicht selten auf unüberwindliche Schwierigkeiten stößt, lassen sich aufgrund der Verbreitung der Expressiva Rückschlüs- 
se auf gemeinsame Elemente in einzelnen ostseefinnischen Sprachen ziehen. Im folgenden soll anhand der Angaben in den Wörterbüchern Suomen kielen etymologinen sanakirja (Etymologisches Wörterbuch des Finnischen, SKES) und Suomen sanojen alkuperä (Die Herkunft der Wörter des Finnischen, SSA) dargelegt werden, inwieweit Entsprechungen der finnischen Expressiva (= deskriptiv-onomatopoetischen Wörter) in den anderen ostseefinnischen Sprachen begegnen. Die im Hinblick auf die Sprecherzahl kleinsten Sprachen, das Wotische und das Livische, mußten (ebenso wie das Ingrische, das im SKES kaum beachtet wurde) wegen des geringen Umfangs des Materials ausgeklammert werden; auch die zwischen dem Karelischen und Wepsischen angesiedelten lüdischen Dialekte wurden nicht gesondert berücksichtigt.

Bekanntlich sind die ersten Bände (I-II) des Etymologischen Wörterbuchs des Finnischen wesentlich knapper gefaßt als die letzten Bände (III-IV), in denen besonderes Augenmerk auf die Darstellung der Bedeutung und Verbreitung der Lexik des Finnischen und seiner nächstverwandten Sprachen gelegt wurde. Es liegt auf der Hand, daß die letzten Bände auch von den Expressiva ein wesentlich vollständigeres Bild zeichnen. Daher gehe ich zunächst auf die Verbreitung der auf $p^{-}, r-, s^{-}, t$ - und $v$ - anlautenden Expressiva ein. Vgl. Diagramme $1-5$, wobei fi. = Finnisch, fiO. = Ostdialekte des Finnischen, kar. $=$ Karelisch, weps. $=$ wepsisch, estn. $=$ Estnisch.

Diagramm 1: Verbreitungsrelationen der auf $p V$ - anlautenden Expressiva im SKES

\begin{tabular}{|c|c|c|}
\hline \multirow{2}{*}{$\begin{array}{l}\text { fi. + kar. } \\
\text { fi. + kar.+ estn. }\end{array}$} & 111 & 56,6 \\
\hline & 23 & 11,7 \\
\hline fi. + kar. + weps. + estn. & 21 & 10,7 \\
\hline fi. + kar. + weps. & 20 & 10,2 \\
\hline fi. + estn. & 12 & 6,1 \\
\hline fi. & 5 & 2,6 \\
\hline Sonstige & 4 & 2,0 \\
\hline Insg. & 196 & 100,0 \\
\hline
\end{tabular}


Diagramm 2: Verbreitungsrelationen der auf $r V$ - anlautenden Expressiva im SKES

fi. + kar. fiO. + kar.

fi. + kar. + weps.

fi. + kar. + estn.

fi. + kar. + weps. + estn.

fi.

fi. + estn.

Sonstige

Insg.

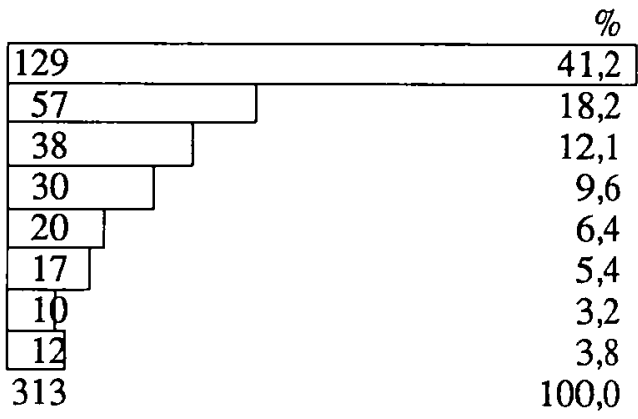

Diagramm 3: Verbreitungsrelationen der auf $s V$ - anlautenden Expressiva im SKES

fi. + kar.

fiO. + kar.

fi. + kar. + weps. + estn.

fi.

fi. + kar. + estn.

fi. + kar. + weps.

fi. + estn.

Sonstige

Insg.

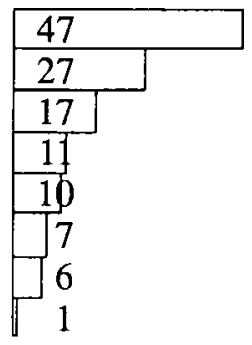

Diagramm 4: Verbreitungsrelationen der auf $t V$ - anlautenden Expressiva im SKES

fi. + kar.

fiO. + kar.

fi. + kar. + estn.

fi. + estn.

fi.

fi. + kar. + weps.

fi. + kar. + weps. + estn.

Sonstige

Insg.

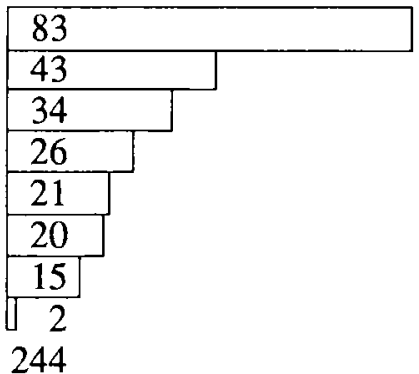

$\%$

33,7

17,5

13,8

10,6

8,5

8,1

6,1

0,8

100,0 
Diagramm 5: Verbreitungsrelationen der auf $v V$ - anlautenden Expressiva im SKES

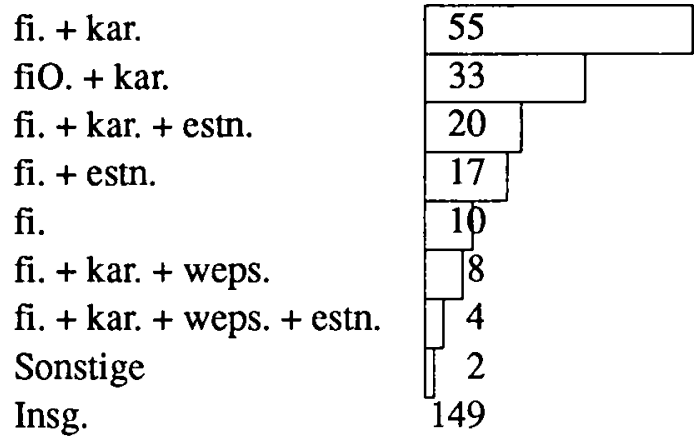

Aus den Diagrammen 1-5 geht bereits auf den ersten Blick hervor, daß im deskriptiv-onomatopoetischen Material des SKES der gemeinsame finnisch-karelische Wortschatz dominiert $(p V-56,6 \%, r V$ $59,4 \%, s V-58,7 \%, t V-51,2 \%$ und $v V-59,0 \%$ ). Eine besonders enge Beziehung scheint $z w i s c h e n$ den Ostdialekten des Finnischen und dem Karelischen zu bestehen: Während so gut wie alle gemeinsamen Wörter der Westdialekte und des Karelischen zugleich auch in den Ostdialekten bekannt sind, gibt es in den östlichen Dialekten und im Karelischen zahlreiche gemeinsame Elemente, die den Westdialekten des Finnischen fremd sind (z. B. $r V-30,6 \%, s V-36,5 \%, t V-34,1 \%$ und $v V$ $37,5 \%$ ). Der Anteil der anderen Verbreitungsgruppen ist in allen oben aufgeführten Fällen gering und beträgt selbst im Höchstfall nur knapp $15 \%$. Insgesamt sind der Gruppe Finnisch + Karelisch + Estnisch 117 Expressiva zuzuordnen (d. h. 11,3\% des gesamten Materials), in der Gruppe Finnisch + Karelisch + Wepsisch beträgt die Gesamtzahl 93 $(8,9 \%)$, in der Gruppe Finnisch + Karelisch + Wepsisch + Estnisch 77 $(7,4 \%)$ und in der Gruppe Finnisch + Estnisch $71(6,8 \%)$; insgesamt 74 Expressiva $(7,1 \%)$ begegnen nur im Finnischen, und die Gruppe "Sonstige" enthält insgesamt 21 Wörter $(2,0 \%)$.

Es erscheint vielleicht überraschend, daß die Anzahl der finnischkarelisch-estnischen Wörter (117) erheblich größer ist als die der finnisch-estnischen. Hierfür gibt es offenbar zwei Gründe: die Größe der 
drei Sprachen und die Kontakte zwischen dem Karelischen und dem Finnischen. Im Hinblick auf die Sprecherzahl sind das Finnische, Karelische und Estnische bei weitem die größten ostseefinnischen Sprachen, und für alle drei Sprachen liegen umfangreiche Wortersammlungen vor; auch der alte gemeinsame Wortschatz blieb zweifellos in diesen Sprachen am besten erhalten. Das Karelische, das dem Finnischen sowohl sprachlich als auch geographisch am nächsten steht, hat aufgrund der engen Kontakte zum Finnischen kontinuierlich starke Impulse von seinem westlichen Nachbarn empfangen.

Auf die gemeinsame ostseefinnische Sprachperiode gehen mit der größten Wahrscheinlichkeit die deskriptiv-onomatopoetischen Wörter und Wortstämme zurück, die nicht nur im Finnischen, Karelischen und Estnischen, sondern auch im Wepsischen bekannt sind. Die Vermutung liegt nahe, daß auch der übrige und vor allem der finnisch-karelischestnische Wortschatz urfinnische Elemente enthält, die in den kleinsten osfi. Sprachen bereits geschwunden sind.

Ein ganz anderes Bild von den Verbreitungsrelationen der deskriptiv-onomatopoetischen Wörter ergibt sich aus dem knapperen Material der ersten Bände des SKES. Dies zeigt beispielsweise die Analyse der auf $h V$ - und $k V$ - anlautenden Wörter, deren Ergebnisse in den Diagrammen 6 und 7 dargestellt werden.

Diagramm 6: Verbreitungsrelationen der auf $h V$ - anlautenden Expressiva im SKES

fi. + estn.

fi. + kar. + estn.

fi. + kar. + weps. + estn.

fi. + kar. + weps.

fi. + kar.

fi.

Sonstige

Insg.

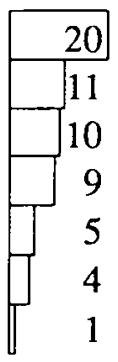

60
$\%$

33,3

18,3

16,7

15,0

8,3

6,7

1,7

100,0 
Diagramm 7: Verbreitungsrelationen der auf $k V$ - anlautenden Expressiva im SKES
fi. + estn.
fi. + kar. + weps. + estn.
fi. + kar. + estn.
fi. + kar. + weps.
fi.
fi. + kar.
Sonstige
Insg.

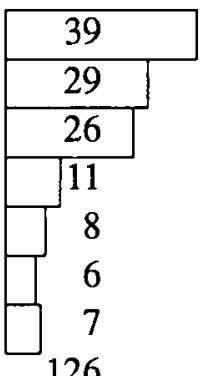

Die Angaben der Diagramme 6 und 7 unterscheiden sich grundsätzlich von den oben (in den Diagrammen 1-5) dargestellten Ergebnissen: Die finnisch-estnischen Wörter bilden hier die größte $(33,3 \%$ und $31,0 \%)$, die finnisch-karelischen die kleinste $(8,3 \%$ und 4,8\%) Gruppe. Die Erklärung ist allerdings einfach: Der Autor des ersten Teils des SKES, Y. H. Toivonen, schenkte den deskriptivonomatopoetischen Wörtern sehr wenig Beachtung und hielt es generell nicht für notwendig, die Verbreitungsrelationen der von ihm etymologisierten Wörter im Einzelnen darzustellen; die damals noch ungeordneten Sammlungen des Wörterbuchs des Karelischen scheint er kaum benutzt zu haben.

Eine Vergleichsmöglichkeit zu den Angaben des Etymologischen Wörterbuchs des Finnischen bietet die grundlegend überarbeitete Neuausgabe des Wörterbuchs, das Werk Suomen sanojen alkuperä (Die Herkunft der Wörter des Finnischen, SSA), dessen erster Band (A-K) kürzlich erschienen ist. Das Material dieses Bandes ergibt für die Verbreitungsrelationen der auf $h V$ und $k V$ - anlautenden Expressiva das folgende Bild (s. Diagramme 8 und 9). 
Diagramm 8: Verbreitungsrelationen der auf $h V$ - anlautenden Expressiva im SSA

fi. + kar.

fi. + kar. + estn.

fi. + kar. + weps.

fi.

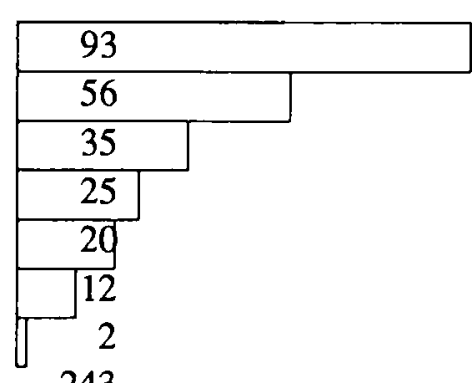

$\%$

38,3

23,0

14,4

10,3

fi. + kar. + weps. + estn.

fi. + estn.

Sonstige

Insg.

243

8,2

4,9

0,8

100,0

Diagramm 9: Verbreitungsrelationen der auf $k V$ - anlautenden Expressiva im SSA

fi. + kar. + estn

fi. + kar.

fi. + kar. + weps. + estn.

fi.

fi. + estn.

fi. + kar. + weps.

Sonstige

Insg.

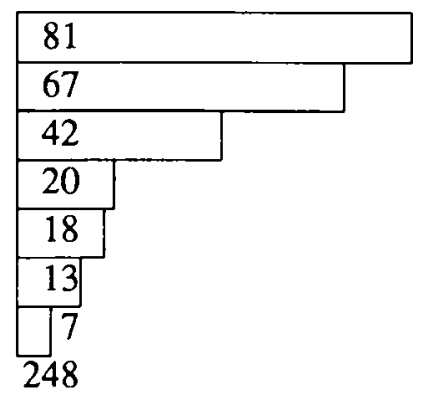

Die Diagramme 8 und 9 zeigen eine völlig andere Konstellation als die Diagramme 6 und 7: Der Anteil der finnisch-estnischen Wörter ist erheblich zurückgegangen (4,9\% und 7,3\%), während der Anteil der finnisch-karelisch-estnischen $(23,0 \%$ und $32,7 \%)$ und vor allem der finnisch-karelischen Wörter (38,3\% und 27,0\%) deutlich gestiegen ist. Die Stellung des finnisch-karelischen Materials ist jedoch weniger dominierend als in den Diagrammen 1-5, die auf den letzten Bänden des SKES beruhen. Dies deutet wohl vor allem auf einen Wandel der Aus- 
wahlkriterien hin: (ost)finnisch-karelische Expressiva, deren Verbreitungsgebiet sehr klein ist, wurden in der Neuausgabe nicht mehr berücksichtigt. Der Hauptgrund für den Anstieg des gemeinsamen finnisch-karelisch-estnischen Wortschatzes wiederum dürfte darin liegen, daß die Angaben über das Estnische in den letzten Jahrzehnten erheblich präzisiert wurden.

Die Diagramme 6-7 und 8-9 zeigen auch einen Anstieg der absoluten Zahlen im Bereich der expressiven Lexik $(h-60 \rightarrow 243, k$ - 126 $\rightarrow 248$ ). Den Einfluß dieser Steigerung auf die Verbreitungsrelationen veranschaulichen die folgenden Zahlen: Unter den auf $h$ - und $k$-anlautenden Wörtern im SSA finden sich in der Gruppe Finnisch + Karelisch 133, in der Gruppe Finnisch + Karelisch + Estnisch 57, in der Gruppe Finnisch + Karelisch + Wepsisch 36 und in der Gruppe Finnisch + Karelisch + Wepsisch + Estnisch 16 Lemmata, die im ersten Teil des Etymologischen Wörterbuchs nicht enthalten sind. Auch die Angaben über die Verbreitung haben sich beträchtlich geändert. So sind etwa 39 auf $h$ - und $k$ - anlautende Wörter, die dem SKES zufolge der Gruppe Finnisch + Estnisch zuzuordnen waren, im SSA in die Gruppe Finnisch + Karelisch + Estnisch eingeordnet worden. Auch in der entgegengesetzten Richtung sind einige Veränderungen zu beobachten; u. a. wird für fünf (5) auf $h$ - oder $k$ - anlautende Wörter, die das SKES der Gruppe Finnisch + Karelisch + Wepsisch + Estnisch zuordnet, im SSA nur das Finnische, Karelische und Estnische als Verbreitungsgebiet angegeben.

\section{Die Anzahl der Expressiva}

Die Verwendung von deskriptiv-onomatopoetischen Wörtern ist selbstverständlich von der Art des Textes abhängig: In der sachbetonten Schriftsprache werden Expressiva so gut wie gar nicht verwendet, während sie in der überwiegend dialogischen gesprochenen Sprache sehr häufig auftreten können. Einleuchtend ist auch, daß die absolute Zahl der in der Schrift- und Hochsprache eingebürgerten Expressiva deutlich niedriger ist als beispielsweise die entsprechende Anzahl in den Dialekten. Die besten Mundartsprecher scheinen tatsächlich die phoneti- 
schen Ausdrucksmöglichkeiten ihrer Sprache in vollem Umfang zu nutzen. Sie begnügen sich nicht damit, allgemein bekannte Expressiva zu variieren, sondern sind gegebenenfalls auch in der Lage, neue deskriptiv-onomatopoetische Ausdrücke zu bilden. Dies trägt dazu bei, die ohnehin umfängliche expressive Lexik der Dialekte weiter zu bereichem. Es ist anzunehmen, daß die Auswirkungen dieser Gegebenheiten auch in den auf verschiedenen Texttypen und Sprachformen beruhenden Materialsammlungen sichtbar werden. Aufschluß darüber gibt Tabelle 1 mit Angaben über die Anzahl und den prozentualen Anteil der Expressiva in fünf verschiedenen Wörterbüchern. Einbezogen wurden neben dem Etymologischen Wörterbuch des Finnischen (SKES) und dem Werk Suomen sanojen alkuperä (SSA) die folgenden Wörterbücher: Nykysuomen sanakirja (Wörterbuch der finnischen Gegenwartssprache, NS), Kiihtelysvaaran murteen sanakirja (Wörterbuch der Mundart von Kiihtelysvaara, KMS) von R. E. Nirvi, das umfangreichste finnische Lexikon der Mundart einer einzelnen Landgemeinde, sowie Karjalan kielen sanakirja (Wörterbuch des Karelischen, KKS 1-3) als Vertreter der dem Finnischen am nächsten verwandten Sprache, die noch keine nennenswerte schriftliche Tradition besitzt.

Tabelle 1: Absolute Zahl und relativer Anteil der Expressiva in fünf Wörterbüchern

\begin{tabular}{|l|c|r|r|}
\hline \multicolumn{2}{|c|}{ Lemmata } & \multicolumn{2}{c|}{ Expressiva } \\
\hline Lexikon & f & \multicolumn{1}{c|}{ f } & $\%$ \\
\hline SKES & 9062 & 1516 & 16,7 \\
SSA 1 & 3507 & 590 & 16,8 \\
NS & 71454 & 5189 & 7,3 \\
KMS & 20904 & 6557 & 31,4 \\
KKS 1-3 & 44315 & 13352 & 30,1 \\
\hline
\end{tabular}

Die Zahlenangaben in Tabelle 1 bestätigen die oben dargelegte Auffassung: Der relative Anteil der Expressiva ist am niedrigsten (nur 7,3\% aller Lemmata) im Wörterbuch der finnischen Gegenwarts- 
sprache, das die Schrift- und Hochsprache repräsentiert; bei weitem den höchsten Anteil an Expressiva enthalten die beiden auf der volkstümlichen gesprochenen Sprache beruhenden Lexika, das Wörterbuch der Mundart von Kiihtelysvaara $(31,4 \%)$ und das Wörterbuch des Karelischen $(30,1 \%)$. Auffällig ist, daß auch die absolute Zahl der deskriptivonomatopoetischen Wörter im Material aus der Mundart einer einzigen Landgemeinde höher ist als im normativen Wörterbuch der gesamten finnischen Sprache. Neben der Textgattung hat vermutlich auch die unterschiedliche Art des Sammelns und der Darstellung des Materials das Ergebnis beeinflußt. Die beiden etymologischen Wörterbücher liegen mit ihren Prozentzahlen (SKES 16,7 \%, SSA 1 16,8 \%) interessanterweise in der Mitte zwischen den Extremwerten. Die Auswahl des Materials dieser beiden Werke wurde in erster Linie durch Forschungsgesichtspunkte bestimmt. Daher mußte auch der für die Dialekte typische deskriptiv-onomatopoetische Wortschatz in die Betrachtung einbezogen werden.

Bei den Expressiva handelt es sich bekanntlich in erster Linie um Substantive und Verben. Der Anteil anderer Wortklassen ist äußerst gering, obwohl sich z. B. viele expressive Verben aus onomatopoetischen Interjektionen entwickelt haben. Einen ungefähren Eindruck von den quantitativen Beziehungen zwischen den Wortklassen vermittelt Tabelle 2, die die expressiven Lemmata im Wörterbuch der finnischen Gegenwartssprache und im Wörterbuch der Mundart von Kiihtelysvaara in ihrer Verteilung auf die einzelnen Wortklassen zeigt.

Tabelle 2: Aufgliederung der Expressiva im NS und im KMS nach Wortklassen

\begin{tabular}{|ll|rrrrrrr|}
\hline $\begin{array}{l}\text { Wörter- } \\
\text { buch }\end{array}$ & Subst. & Adj. & Verb & Adv. & Interj. & Sonst. & Insg. \\
\hline NS & f & 813 & 146 & 3921 & 76 & 170 & 63 & 5189 \\
& $\%$ & 15,7 & 2,8 & 75,6 & 1,5 & 3,3 & 1,2 & 100,0 \\
KMS & f & 1541 & 369 & 4079 & 124 & 6 & 438 & 6557 \\
& $\%$ & 23,5 & 5,6 & 62,2 & 1,9 & 0,1 & 6,7 & 100,0 \\
\hline
\end{tabular}


In beiden Korpora der Tabelle 2 bilden die Verben eindeutig die dominierende Wortklasse. Ihr relativer Anteil liegt jedoch im NS wesentlich höher als im KMS; die Differenz ist mit 13,4 Prozentpunkten beträchtlich. Andererseits enthält das NS erheblich weniger expressive Substantive als das KMS. Beides ist wohl einerseits auf das unterschiedliche Material (Schriftsprache contra gesprochene Sprache), andererseits auf die jeweilige Art der Auswahl und Darstellung des Materials zurückzuführen. Auch die geringe Anzahl der Interjektionen im KMS ist dadurch zu erklären. Der Grund für den hohen Anteil unklassifizierter Expressiva im KMS (6,7 \%) liegt darin, daß der Autor homonyme Substantive und Adjektive in einer Gruppe, als Nomina, zusammengefaßt hat.

\section{Phonologie und Phonotaktik}

Im Hinblick auf die Lautstruktur besteht bekanntlich zwischen der expressiven und der sonstigen, nicht affektiven Lexik einer Sprache keine vollständige Übereinstimmung. Vor allem die Onomatopoetika bevorzugen seltene Laute und ungewöhnliche Lautkombinationen. Im Fall des Finnischen wurde schon früh u. a. festgestellt, daß der Tremulant $r$ und der Frikativ $h$ hohe onomatopoetische Ausdruckskraft besitzen und daß der Vokal $\ddot{o}$ stark affektiv gefärbt ist. Auch die Nasale $m$ und $n$ sowie die Liquida $l$ treten in Onomatopoetika überdurchschnittlich häufig auf. Da diese Eigenheiten offenbar allen ostseefinnischen Sprachen gemeinsam sind, müßten sie auch im expressiven Material der etymologischen Wörterbücher zutage treten. Zur Überprüfung dieser Annahme wurden in den folgenden Tabellen Angaben über die Frequenzrelationen von in verschiedener Position auftretenden Konsonanten und Vokalen sowie von einigen Lautverbindungen in den Lemmata des SKES und des SSA zusammengestellt.

Schon die erste Tabelle (3a), die den Anteil der Expressiva an den konsonantisch anlautenden Lemmata des SKES zeigt, verrät die Unausgewogenheit des Materials: Im Bereich $h-n$ ist die Zahl der Expressiva wesentlich geringer als im Bereich $p-v$. In der zweiten Hälfte des 
Werkes ist besonders der Anteil der Expressiva deutlich gestiegen. Dies gilt auch für die neue Fassung des Wörterbuchs: Das SSA enthält im Bereich $h-k 361$ expressive Lemmata mehr als der entsprechende Abschnitt des SKES. Bei der Interpretation der numerischen Angaben der folgenden Tabellen ist dies natürlich zu berücksichtigen.

Tabelle 3a: Der Anteil der Expressiva an den auf CanlautendenLemmata des SKES

\begin{tabular}{|l|r|r|r|}
\hline \multicolumn{2}{|c|}{ Lemmata } & \multicolumn{2}{|c|}{ Expressiva } \\
$C-$ & $\mathrm{f}$ & $\mathrm{f}$ & $\%$ \\
\hline$h-$ & 473 & 60 & 12,7 \\
$j-$ & 144 & 15 & 10,4 \\
$k-$ & 1135 & 126 & 11,1 \\
$l-$ & 551 & 67 & 12,2 \\
$m-$ & 345 & 43 & 12,5 \\
$n-$ & 369 & 95 & 25,7 \\
$p-$ & 1130 & 196 & 17,3 \\
$r-$ & 1051 & 313 & 29,8 \\
$s-$ & 923 & 136 & 14,7 \\
$t-$ & 1085 & 246 & 22,7 \\
$v-$ & 950 & 149 & 15,7 \\
Insg. & 8156 & 1446 & 17,7 \\
\hline
\end{tabular}

Tabelle 4a: Der Anteil der Expressiva an den auf $\mathrm{V}$ anlautenden Lemmata des SKES

\begin{tabular}{|l|r|r|r|}
\hline \multicolumn{2}{|c|}{ Lemmata } & \multicolumn{2}{c|}{ Expressiva } \\
C- & $\mathrm{f}$ & $\mathrm{f}$ & $\%$ \\
\hline$a-$ & 225 & 6 & 2,7 \\
$e-$ & 51 & - & 0,0 \\
$i-$ & 60 & 5 & 8,3 \\
$o-$ & 85 & 2 & 2,4 \\
$u-$ & 167 & 20 & 12,0 \\
$y-$ & 21 & 3 & 14,3 \\
$\ddot{a}-$ & 65 & 21 & 32,3 \\
$\ddot{o}-$ & 19 & 10 & 52,6 \\
Insg. & 693 & 67 & 9,7 \\
\hline
\end{tabular}

Tabelle 3b: Der Anteil der Expressiva an den auf Canlautenden Lemmata des SSA

\begin{tabular}{|l|r|r|r|}
\hline \multicolumn{2}{|c|}{ Lemmata } & \multicolumn{2}{c|}{ Expressiva } \\
C- & $\mathrm{f}$ & $\mathrm{f}$ & $\%$ \\
\hline$h-$ & 776 & 248 & 32,0 \\
$j-$ & 244 & 54 & 22,1 \\
$k-$ & 1642 & 260 & 15,8 \\
Insg. & 2662 & 562 & 21,1 \\
\hline
\end{tabular}

Tabelle 4b: Der Anteil der Expressiva an den auf Vanlautenden Lemmata des SSA

\begin{tabular}{|l|r|r|r|}
\hline \multicolumn{3}{|c|}{ Lemmata } & \multicolumn{2}{|c|}{ Expressiva } \\
C- & f & f & $\%$ \\
\hline$a-$ & 355 & 10 & 2,8 \\
$e-$ & 82 & - & 0,0 \\
$i-$ & 78 & 5 & 6,4 \\
Insg. & 515 & 15 & 2,9 \\
\hline
\end{tabular}


Die Angaben der Tabellen 3a und 3b vermitteln nur ein ungefähres Bild der Rolle und Bedeutung der einzelnen anlautenden Konsonanten als Indikatoren der Expressivität, denn der geringere Umfang des Materials in der ersten Hälfte des SKES und die Tatsache, daß das SSA noch nicht vollständig vorliegt, verhindem gesicherte Schlußfolgerungen. Im Material des SKES ist jedenfalls der relative Anteil der auf $r$ $(29,8 \%), n-(25,7 \%)$ und $t-(22,7 \%)$ anlautenden deskriptiv-onomatopoetischen Wörter am höchsten, der Anteil der auf $j$ - (10,4\%) und $k$ $(11,1 \%)$ anlautenden am niedrigsten. Der prozentuale Anteil der anderen Konsonanten der ersten Hälfte des Alphabets ist nahezu genauso niedrig, während die Konsonanten der letzten Hälfte des Alphabets erwartungsgemäB deutlich höhere Prozentzahlen aufweisen (NB. $p$ $17,3 \%, v-15,7 \%$ und $s-14,7 \%)$. Besonders auffällig ist der geringe Anteil $(12,7 \%)$ der auf $h$ - anlautenden Expressiva im SKES, steht doch dieser Befund im Widerspruch zu der oben erwähnten allgemeinen Beobachtung. Auch diese Prozentzahl erweist sich als auf den geringen Umfang des Materials zurückzuführendes Trugbild, wenn man sie mit den Angaben zum SSA vergleicht: Diesen zufolge liegt der Anteil des deskriptiv-onomatopoetischen Materials an den auf $h$ - anlautenden Lemmata bei 32,0\% und ist zugleich bedeutend höher als bei den auf $j$ und $k$ - anlautenden Lemmata (22,1\% und 15,8\%). Das Beispiel macht zudem deutlich, welche Fülle von Expressiva in die neue Fassung des etymologischen Wörterbuchs aufgenommen wurde.

Aus den Tabellen $4 \mathrm{a}$ und $4 \mathrm{~b}$, die das vokalisch anlautende Material aufgliedern, geht bereits auf den ersten Blick hervor, daß das Korpus insgesamt wenig umfangreich ist und daß zudem auch der relative Anteil der vokalisch anlautenden Expressiva wesentlich niedriger ist als der der konsonantisch anlautenden. Bemerkenswert ist ferner, daß der Schwerpunkt des expressiven Materials auf den Vokalen der zweiten Hälfte des Alphabets liegt. Obwohl die Vokale $y, \ddot{a}$ und $\ddot{o}$ im normalen Wortschatz die niedrigste Frequenz aufweisen, sind sie in der deskriptiv-onomatopoetischen Lexik sowohl relativ als auch absolut die frequentesten Vokale. Dies gilt ganz besonders für den Vokal $\ddot{o}$ : 10 der 19 auf $\ddot{o}$ - anlautenden Lemmata im SKES sind deskriptiv-onomatopoetische Wörter; bei den anderen 9 handelt es sich um jüngere Entlehnungen aus dem Schwedischen. - Es sei ferner besonders darauf hinge- 
wiesen, daß das Material keine auf $e$ - anlautende Expressiva enthält und da $\beta$ auch die Anzahl der auf $a$ - und $i$ - anlautenden Expressiva überraschend niedrig ist.

Über die regionale Verteilung der verschiedenen CV-Typen lassen sich aufgrund des geringen Umfangs des Materials keine gesicherten Aussagen machen. Offensichtlich sind jedoch die auf $C \ddot{o}$ - anlautenden Expressiva ihrer Verbreitung nach noch deutlicher finnisch-karelisch oder finnisch als das übrige Material. Siehe dazu Tabelle 5 über die Verbreitungsrelationen der auf $p \ddot{p}-$, rö- und $t \ddot{o}$ - anlautenden Expressiva im SKES.

Tabelle 5: Regionale Gliederung der auf pö-, rö- und töanlautenden Expressiva im SKES

\begin{tabular}{|l|rrr|r|r|}
\hline Verbreitung & $p \ddot{o}-$ & $r \ddot{o}-$ & $t \ddot{o}-$ & Insg. & $\%$ \\
\hline fi. + kar. & 23 & 14 & 27 & 64 & 51,2 \\
fiO. + kar. & 8 & 7 & 19 & 34 & 27,2 \\
fi. + kar. + weps. & 4 & 3 & 4 & 11 & 8,8 \\
fi. + kar. + estn. & - & 2 & 3 & 5 & 4,0 \\
fi. & - & 2 & 3 & 5 & 4,0 \\
fi. + kar. + weps. + estn. & 2 & 1 & - & 3 & 2,4 \\
fi. + estn. & - & - & 1 & 1 & 0,8 \\
Sonstige & 1 & 1 & - & 2 & 1,6 \\
\hline Insgesamt & 38 & 30 & 57 & 125 & 100,0 \\
\hline
\end{tabular}

Wie die Tabelle zeigt, handelt es sich bei 103 der auf pö-, rö- oder tö- anlautenden Expressiva, also bei 84,4 \% der Gesamtzahl (125) um finnisch-karelische oder finnische Wörter. Der Anteil dieser beiden Verbreitungsgruppen an allen auf $p V$ - anlautenden Expressiva beträgt nur 59,2\%, bei anlautendem $r V-64,8 \%$ und bei anlautendem $t V$ $59,7 \%$. Dies ist ein weiterer Hinweis darauf, daß die finnischen Expressiva, in denen der Vokal -ö- auftritt, verhältnismäßig spät entstanden sind.

Zum Vergleich seien Angaben über Zahl und Lautstruktur der Expressiva in zwei finnischen Wörterbüchem angeführt, die sich beide durch Materialfülle und relativ gleichmäßigen Aufbau auszeichnen: a) das Wörterbuch der finnischen Gegenwartssprache (NS), ein Lexikon der Schrift- und Hochsprache, und b) das bisher umfangreichste Lexikon der Mundart einer einzelnen Landgemeinde, das Wörterbuch 
der Mundart von Kiihtelysvaara (KMS) von R. E. Nirvi, das die sog. östlichen Dialekte von Savo vertritt. - Siehe Tabellen $6 \mathrm{a}$ und $6 \mathrm{~b}$ sowie $7 \mathrm{a}$ und $7 \mathrm{~b}$.

Tabelle 6a: Der Anteil der

Expressiva an den auf $\mathrm{C}$ -

anlautenden Lemmata des NS

\begin{tabular}{|l|r|r|r|}
\hline \multicolumn{3}{|r|}{ Lemmata } & \multicolumn{2}{|r|}{ Expressiva } \\
C- & f & f & $\%$ \\
\hline$h-$ & 5906 & 672 & 11,4 \\
$j-$ & 1822 & 235 & 12,9 \\
$k-$ & 10645 & 958 & 9,0 \\
$l-$ & 5302 & 607 & 11,5 \\
$m-$ & 3907 & 319 & 8,2 \\
$n-$ & 2673 & 342 & 12,8 \\
$p-$ & 7464 & 393 & 5,8 \\
$r-$ & 4650 & 672 & 14,5 \\
$s-$ & 6662 & 187 & 2,8 \\
$t-$ & 6256 & 476 & 7,6 \\
$v-$ & 5187 & 127 & 2,5 \\
\hline Insg. & 60474 & 4988 & 8,2 \\
\hline
\end{tabular}

Tabelle 7a: Der Anteil der

Expressiva an den auf V-

anlautenden Lemmata des NS

\begin{tabular}{|l|r|r|r|}
\hline \multicolumn{2}{|c|}{ Lemmata } & \multicolumn{2}{|c|}{ Expressiva } \\
$\mathrm{C}-$ & $\mathrm{f}$ & $\mathrm{f}$ & $\%$ \\
\hline$a-$ & 2697 & 20 & 0,7 \\
$e-$ & 1451 & - & 0,0 \\
$i-$ & 1403 & 14 & 1,0 \\
$o-$ & 1287 & 10 & 0,8 \\
$u-$ & 876 & 34 & 3,9 \\
$y-$ & 578 & 11 & 1,9 \\
$\ddot{a}-$ & 518 & 94 & 18,2 \\
$\ddot{o}-$ & 73 & 23 & 31,5 \\
\hline Insg. & 8883 & 206 & 2,3 \\
\hline
\end{tabular}

Tabelle 6b: Der Anteil der Expressiva an den auf $\mathrm{C}$ anlautenden Lemmata des KMS

\begin{tabular}{|l|r|r|r|}
\hline \multicolumn{2}{|r|}{ Lemmata } & \multicolumn{2}{r|}{ Expressiva } \\
C- & f & f & $\%$ \\
\hline$h-$ & 1707 & 693 & 40,6 \\
$j-$ & 718 & 270 & 37,6 \\
$k-$ & 3484 & 1116 & 32,0 \\
$l-$ & 1824 & 709 & 38,9 \\
$m-$ & 1058 & 360 & 34,0 \\
$n-$ & 819 & 292 & 35,7 \\
$p-$ & 2150 & 483 & 22,5 \\
$r-$ & 1970 & 1045 & 53,0 \\
$s-$ & 1694 & 378 & 22,3 \\
$t-$ & 1711 & 544 & 31,8 \\
$\nu-$ & 1849 & 383 & 20,7 \\
\hline Insg. & 18984 & 6273 & 33,0 \\
\hline
\end{tabular}

Tabelle 7b: Der Anteil der Expressiva an den auf Vanlautenden Lemmata des KMS

\begin{tabular}{|l|r|r|r|}
\hline \multicolumn{2}{|r|}{ Lemmata } & \multicolumn{2}{r|}{ Expressiva } \\
C- & f & f & $\%$ \\
\hline$a-$ & 386 & 5 & 1,3 \\
$e-$ & 247 & 5 & 2,0 \\
$i-$ & 246 & 25 & 10,2 \\
$o-$ & 212 & 3 & 1,4 \\
$u-$ & 264 & 35 & 13,3 \\
$y-$ & 226 & 10 & 4,4 \\
$\ddot{a}-$ & 252 & 137 & 54,4 \\
$\ddot{o}-$ & 77 & 64 & 83,1 \\
\hline Insg. & 1910 & 284 & 14,9 \\
\hline
\end{tabular}

Diese beiden Wörterbücher scheinen sich sehr stark voneinander zu unterscheiden: Der Gesamtanteil der Expressiva liegt im NS bei 8,2 \% 
und $2,3 \%$, im KMS bei $33,0 \%$ und $14,9 \%$. Die Zahlen veranschaulichen eindrucksvoll den Unterschied zwischen der normativen Schriftsprache und dem alten Dialekt. Die erstere scheint geradezu vor deskriptiv-onomatopoetischen Ausdrücken zurückzuscheuen, während der letztere eine deutliche Vorliebe für Expressiva zeigt. Dennoch ergibt sich aus beiden Quellen für die Anlaute der Expressiva im Hinblick auf ihre relative Häufigkeit eine ähnliche Reihenfolge. Bei den Konsonanten stehen im NS $r-(14,5 \%), j-(12,9 \%), n-(12,8 \%), l-(11,5 \%)$ und $h$ an der Spitze, im KMS $r-(53 \%), h-(40,6 \%), l-(38,9 \%), j-(37,6 \%)$ und $n$ - $(35,7 \%)$. Die niedrigste relative Frequenz ergibt sich aus beiden Korpora für die Konsonanten $t$ - $(7,6 \%$ und $31,8 \%), p$ - $(5,8 \%$ und $22,5 \%)$, $s-(2,8 \%$ und $22,3 \%)$ und $v-(2,5 \%$ und $20,7 \%)$. Zwischen diesen beiden Gruppen ordnen sich $k$ - und $m$ - ein (NS $k$ - 9,0\% und $m$ - 8,2\%; KMS $m-34 \%$ und $k-32 \%$ ). Die Reihenfolge der Vokale ist in beiden Quellen zunächst gleich: $\ddot{o}$ - (NS 31,5\%, KMS 83,1\%!), $\ddot{a}$ - und $u$-. Es folgen $y$ - und $i$-, im NS in dieser, im KMS in umgekehrter Reihenfolge. Die anderen Vokale begegnen im Anlaut von Expressiva äußerst selten.

Für die Tabellen $8 \mathrm{a}$ und $8 \mathrm{~b}$ wurde das mit einer CV-Verbindung anlautende Material von SKES und SSA nach dem Vokal der ersten Silbe gruppiert, um festzustellen, welche inlautenden Vokale für Expressiva charakteristisch sind. Das Vergleichsmaterial aus NS und KMS wird in den Tabellen $9 \mathrm{a}$ und $9 \mathrm{~b}$ präsentiert.

Tabelle 8a: Der Anteil der Expressiva an den auf CV-anlautenden Lemmata des SKES, klassifiziert nach dem Vokal der ersten Silbe

\begin{tabular}{|l|r|r|r|}
\hline \multicolumn{2}{|c|}{ Lemmata } & \multicolumn{2}{|c|}{ Expressiva } \\
$\mathrm{C}-$ & $\mathrm{f}$ & $\mathrm{f}$ & $\%$ \\
\hline $\mathrm{Ca}-$ & 2184 & 178 & 8,2 \\
$\mathrm{Ce}-$ & 813 & 92 & 11,3 \\
$\mathrm{Ci}-$ & 1454 & 290 & 19,9 \\
$\mathrm{Co}-$ & 1019 & 207 & 20,3 \\
$\mathrm{Cu}-$ & 1395 & 257 & 18,4 \\
$\mathrm{Cy}-$ & 407 & 98 & 24,1 \\
$\mathrm{Cä}-$ & 640 & 166 & 25,9 \\
$\mathrm{Cö}-$ & 244 & 158 & 64,8 \\
\hline Insg. & 8156 & 1446 & 17,7 \\
\hline
\end{tabular}

Tabelle 8b: Der Anteil der Expressiva an den auf CVanlautenden Lemmata des SSA, klassifiziert nach dem Vokal der ersten Silbe

\begin{tabular}{|l|r|r|r|}
\hline \multicolumn{3}{|r|}{ Lemmata } & \multicolumn{3}{|c|}{ Expressiva } \\
$\mathrm{CV}-$ & $\mathrm{f}$ & $\mathrm{f}$ & $\%$ \\
\hline $\mathrm{Ca}-$ & 784 & 107 & 13,6 \\
$\mathrm{Ce}-$ & 221 & 23 & 10,4 \\
$\mathrm{Ci}-$ & 315 & 68 & 21,6 \\
$\mathrm{Co}-$ & 411 & 80 & 29,5 \\
$\mathrm{Cu}-$ & 514 & 114 & 22,2 \\
$\mathrm{Cy}-$ & 138 & 51 & 37,0 \\
$\mathrm{Cä}-$ & 217 & 77 & 35,5 \\
$\mathrm{Cö}-$ & 62 & 42 & 67,7 \\
\hline Insg. & 2662 & 562 & 21,1 \\
\hline
\end{tabular}


Tabelle 9a: Der Anteil der Expressiva an den auf CV-anlautenden Lemmata des NS, klassifiziert nach dem Vokal der ersten Silbe

\begin{tabular}{|l|r|r|r|}
\hline & \multicolumn{2}{|r|}{ Lemmata } & \multicolumn{2}{|c|}{ Expressiva } \\
$\mathrm{CV}--$ & $\mathrm{f}$ & $\mathrm{f}$ & $\%$ \\
\hline $\mathrm{Ca}-$ & 14035 & 590 & 4,2 \\
$\mathrm{Ce}-$ & 7514 & 440 & 5,9 \\
$\mathrm{C} i-$ & 9739 & 754 & 7,7 \\
$\mathrm{Co}-$ & 7387 & 819 & 11,1 \\
$\mathrm{Cu}-$ & 9628 & 887 & 9,2 \\
$\mathrm{Cy}-$ & 3899 & 414 & 10,6 \\
$\mathrm{Cä}-$ & 4660 & 528 & 11,3 \\
$\mathrm{Cö}-$ & 1636 & 526 & 32,2 \\
\hline Insg. & 58498 & 4958 & 8,5 \\
\hline
\end{tabular}

Tabelle 9b: Der Anteil der Expressiva an den auf $\mathrm{CV}$ - anlautenden Lemmata des KMS,klassifiziert nach dem Vokal der ersten Silbe

\begin{tabular}{|l|r|r|r|}
\hline \multicolumn{2}{|l|}{ Lemmata } & \multicolumn{2}{|c|}{ Expressiva } \\
$\mathrm{CV}-$ & $\mathrm{f}$ & $\mathrm{f}$ & $\%$ \\
\hline $\mathrm{Ca}-$ & 4073 & 718 & 17,6 \\
$\mathrm{Ce}-$ & 2062 & 566 & 27,4 \\
$\mathrm{Ci}-$ & 3196 & 907 & 28,4 \\
$\mathrm{Co}-$ & 2265 & 915 & 40,4 \\
$\mathrm{Cu}-$ & 3493 & 1252 & 35,8 \\
$\mathrm{Cy}-$ & 1269 & 528 & 41,6 \\
$\mathrm{Cä}-$ & 1665 & 602 & 36,2 \\
$\mathrm{Cö}-$ & 961 & 785 & 81,7 \\
\hline Insg. & 18984 & 6273 & 33,0 \\
\hline
\end{tabular}

Obwohl die Korpora des SKES und des SSA von sehr unterschiedlichem Umfang sind und zum Teil nach divergenten Kriterien ausgewählt wurden, zeigen die Ergebnisse der Tabellen 8a und 8b eine übereinstimmende Tendenz: SKES $\ddot{o}, \ddot{a}, y, o, i, u, e, a, \operatorname{SSA} \ddot{o}, y$, $\ddot{a}, u, i, o, a, e, \mathrm{~d} . \mathrm{h}$. die in der zweiten Hälfte des Alphabets stehenden Vokale dominieren, während die am Anfang des Alphabets stehenden am wenigsten verbreitet sind. Das gleiche Bild zeigen auch die beiden zum Vergleich herangezogenen Korpora (Tabelle 9a und $9 \mathrm{~b})$. Sie scheinen sich sogar näher zu stehen als SKES und SSA (NS $\ddot{o}, \ddot{a}, o, y, u, i, e, a$; KMS $\ddot{o}, y, o, \ddot{a}, u, i, e, a)$. Besonders auffallend sind die genaue Übereinstimmung am Ende der Liste und die Stellung des $o$ als dritthäufigster Vokal der ersten Silbe in der expressiven Lexik.

Das in den Tabellen 8-9 gezeichnete Bild von der Häufigkeit der wortanlautenden CV-Sequenzen ist insofem unvollständig, als dabei nur der Wechsel des Vokals der ersten Silbe berücksichtigt wurde; der Einfluß des Konsonanten (oder der gemeinsame Einfluß von Konsonant und Vokal) auf die Häufigkeit der einzelnen CVSequenzen wurde dagegen nicht beachtet. Da eine detaillierte Darstellung des gesamten Materials in diesem Rahmen nicht möglich ist, beschränke ich mich darauf, im folgenden die auffälligsten Abweichungen zu nennen: 
- Die Frequenz der auf -ha anlautenden Expressiva liegt in allen Teilkorpora deutlich unter dem Durchschnitt (SKES 2,7 \%, SSA 7,6 $\%$, NS 3,3\%, KMS 17,9\%).

- Das Material enthält keine auf $j i$ - anlautenden Expressiva, und auch je- im Anlaut begegnet nur im KMS. Dagegen ist die relative Frequenz der auf $j y$ - anlautenden Expressiva sehr hoch (SKES 50,0 $\%$, SSA $57,9 \%$, NS 41,4\%, KMS 62,9\%).

- Die Häufigkeit der auf la- anlautenden Expressiva liegt unter dem Durchschnitt (SKES 5,6 \%, NS 3,5\%, KMS 15,5\%), während loim Anlaut sehr häufig auftritt (SKES 21,5\%, NS 22,1\%, KMS $55,4 \%)$.

- Auf $m e$ - und besonders auf $m i$ - anlautende Expressiva sind äußerst selten (SKES 2,5\% und 0,0 \%, NS 1,9\% und 0,0\%, KMS 13,1\% und $7,3 \%)$.

- Auf ne- anlautende Expressiva treten kaum auf (SKES 0,0\%, NS $1,2 \%$, KMS 3,6 \% aller Lemmata auf ne-).

- Der relative Anteil der auf pa- und pe- anlautenden Expressiva liegt in allen Teilkorpora deutlich unter dem Durchschnitt (SKES 7,7\% und $9,5 \%$, NS $1,4 \%$ und $1,2 \%$, KMS $15,4 \%$ und $12,0 \%$ ).

- Die Sequenzen sa- und se- begegnen im Anlaut der Expressiva äußerst selten (SKES 3,0 und 2,3\%, NS 0,0\% und 0,1\%, KMS $5,0 \%$ und $5,2 \%$ ).

- Auch auf $t a$ - und $t \ddot{a}$ - anlautende Expressiva sind wenig verbreitet (SKES 7,2 \% und $12,5 \%$, NS $1,8 \%$ und $2,9 \%$, KMS 8,5 \% und $13,0 \%)$.

- Im Anlaut von Expressiva begegnet $v a$ - selten (SKES 5,4 \%, NS $0,3 \%$, KMS 6,1\%), vo- ist überdurchschnittlich frequent (SKES $19,6 \%$, NS $13,5 \%$, KMS $60,9 \%$ ), während $v u$ - ebenfalls selten verwendet wird (SKES 7,0 \%, NS 0,6\%, KMS 12,3\%).

Es ist schwierig, spezielle Gründe für die unterschiedliche Häufigkeit der Lautverbindungen aufzuzeigen. Das seltene Auftreten der Sequenzen $j e-, j i$ - und $v u$ - ist offensichtlich darauf zurückzuführen, daß sich die Konsonanten $j$ - und $v$ - im allgemeinen nicht mit den ihnen phonetisch am nächsten stehenden Vokalen verbinden (Wiik 1969). Die Sequenz $\mathrm{Ca}$-wiederum wird in Expressiva wohl deshalb selten verwendet, weil sie im normalen Wortschatz sehr häufig auftritt und kaum 
affektiv gefärbt zu sein scheint. Dagegen läßt sich die geringe Frequenz der Verbindungen $m e-$, $m i$ - und $n e$ - nicht durch den Aspekt der Farblosigkeit erklären.

Um die Häufigkeit der verschiedenen inlautenden Konsonanten zu ermitteln, wurden in Tabelle 10 Angaben über die Lemmata mit der Struktur CV(V)CV- in dem Herkunftswörterbuch Suomen sanojen alkuperä zusammengestellt. Die Tabelle enthält folgende Informationen: a) die Gesamtzahl der Wörter, b) die Anzahl der Expressiva, c) der prozentuale Anteil der Expressiva sowie d) die entsprechenden Angaben für die nach dem inlautenden Konsonant gegliederten Subkorpora.

Tabelle 10: Der Anteil der Expressiva an den auf CV(V)CVanlautenden Lemmata des SSA, gruppiert nach dem inlautenden Konsonanten

\begin{tabular}{|l|r|r|r|}
\hline \multicolumn{2}{|c|}{ Lemmata } & \multicolumn{2}{c|}{ Expressiva } \\
$\mathrm{CV}(\mathrm{V}) \mathrm{CV}$ & $\mathrm{f}$ & $\mathrm{f}$ & $\%$ \\
\hline$-h-$ & 48 & 22 & 45,8 \\
$-j-$ & 23 & 6 & 26,1 \\
$-k-$ & 101 & 33 & 32,7 \\
$-l-$ & 145 & 37 & 25,5 \\
$-m-$ & 116 & 41 & 35,3 \\
$-n-$ & 60 & 7 & 11,7 \\
$-p-$ & 105 & 40 & 38,1 \\
$-r-$ & 186 & 48 & 25,8 \\
$-s-$ & 89 & 6 & 6,7 \\
$-t-$ & 111 & 24 & 21,6 \\
$-\nu-$ & 82 & 7 & 8,5 \\
\hline Insg. & 1066 & 271 & 25,4 \\
\hline
\end{tabular}

Zwar beruht Tabelle 10 auf einem wenig umfangreichen Korpus (1066 Lemmata), doch läßt sich aus den Ergebnissen zumindest eine bestimmte Tendenz ablesen. Als inlautender Konsonant nach der hauptbetonten Silbe begegnet demnach in der expressiven Lexik besonders häufig $-h$ - $(45,8 \%$ aller Lemmata dieser Gruppe), $-p$ - $(38,1 \%),-m$ $(35,3 \%)$ und $-k$ - $(32,7 \%)$, während $-s-(6,7 \%),-v-(8,5 \%)$ und $-n-$ $(11,7 \%)$ die geringste Frequenz aufweisen. 
Entsprechende Angaben für Geminaten und Verbindungen von zwei verschiedenen Konsonanten zwischen erster und zweiter Silbe sind in Tabelle 11 zusammengefaßt. Das Material stammt ebenfalls aus dem ersten Band $(a-k)$ des Werkes Suomen sanojen alkuperä.

Tabelle 11: Die Frequenz der an der Grenze zwischen erster und zweiter Silbe auftretenden Geminaten und Konsonantenverbindungen ( $=\mathrm{C}(\mathrm{C}) \mathrm{C}$-Sequenzen) in den expressiven Lemmata des SSA

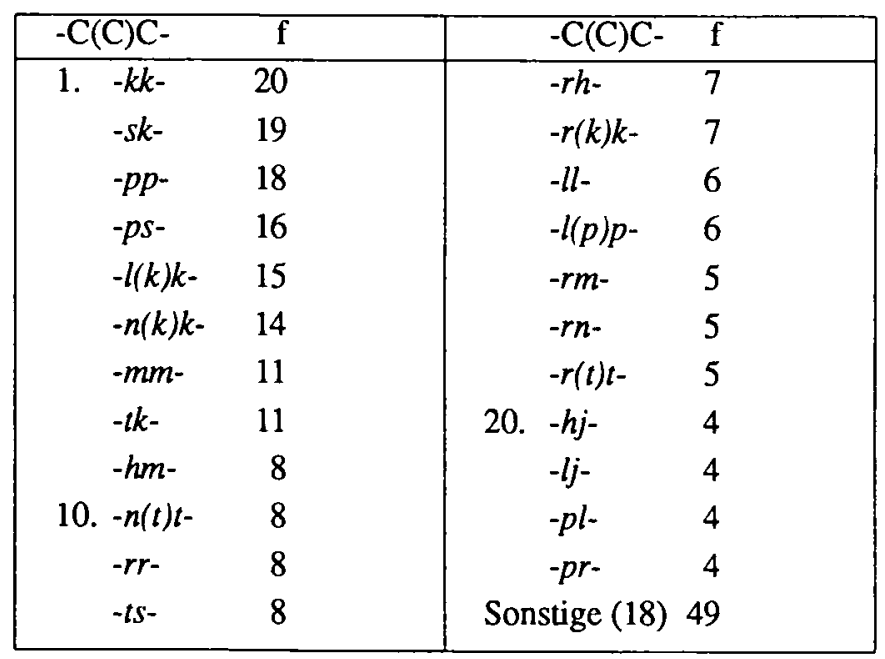

Das Korpus enthält insgesamt $286 \mathrm{C}(\mathrm{C}) \mathrm{C}$-Sequenzen, die sich auf 41 verschiedene Verbindungen verteilen (8 Geminaten und 33 andere Konsonantenverbindungen). Da das Material in eine Vielzahl von Gruppen zerfällt, ist es nicht umfangreich genug, um ein zuverlässiges Bild von den Häufigkeitsrelationen der einzelnen $\mathrm{C}(\mathrm{C}) \mathrm{C}$-Sequenzen $\mathrm{zu}$ vermitteln. Deshalb werden in den Tabellen $12 \mathrm{a}$ und $12 \mathrm{~b}$ die entsprechenden Angaben zur Frequenz der Konsonantenverbindungen in den expressiven Lemmata des NS und des KMS dargestellt. 
Tabelle 12a: Die absolute und prozentuale Frequenz der an der Grenze zwischen erster und zweiter Silbe auftretenden Konsonantenverbindungen im NS

\begin{tabular}{|c|c|c|c|c|c|c|c|}
\hline & $-C(C) C-$ & $\mathrm{f}$ & $\%$ & & $-C(C) C-$ & $\mathrm{f}$ & $\%$ \\
\hline \multirow[t]{9}{*}{1.} & $-s k-$ & 450 & 23,3 & & $-h r-$ & 16 & 0,8 \\
\hline & $-p s-$ & 248 & 12,8 & & $-t j$ & 16 & 0,8 \\
\hline & $-t k-$ & 166 & 8,6 & & $-r j-$ & 14 & 0,7 \\
\hline & $-h k-$ & 113 & 5,9 & & $-r m-$ & 14 & 0,7 \\
\hline & $-k s-$ & 107 & 5,5 & & $-r v-$ & 14 & 0,7 \\
\hline & $-l(k) k-$ & 93 & 4,8 & & $-t s-$ & 10 & 0,5 \\
\hline & $-n(k) k-$ & 86 & 4,5 & 30. & $-h t-$ & 9 & 0,5 \\
\hline & $-l j-$ & 59 & 3,1 & & $-s t-$ & 8 & 0,4 \\
\hline & $-m(p) p$ & 59 & 3,1 & & $-h l-$ & 7 & 0,4 \\
\hline & $-n(t) t-$ & 50 & 2,6 & & $-k l-$ & 7 & 0,4 \\
\hline & $-h n-$ & 48 & 2,6 & & $-t r$ & 6 & 0,3 \\
\hline & $-h j-$ & 39 & 2,0 & & $-h v-$ & 3 & 0,2 \\
\hline & $-r(p) p$ & 35 & 1,8 & & $-r l-$ & 3 & 0,2 \\
\hline & $-h m-$ & 31 & 1,6 & & $-r(t) t-$ & 3 & 0,2 \\
\hline & $-r h-$ & 27 & 1,4 & & $-t v-$ & 3 & 0,2 \\
\hline & $-l(p) p$ & 25 & 1,3 & & $-l h-$ & 2 & 0,1 \\
\hline & $-p l-$ & 25 & 1,3 & 40. & $-t l-$ & 2 & 0,1 \\
\hline & $-l m-$ & 24 & 1,2 & & $-l s-$ & 1 & 0,1 \\
\hline & $-r n-$ & 24 & 1,2 & & $-n s-$ & 1 & 0,1 \\
\hline \multirow[t]{5}{*}{20 . } & $-p r-$ & 23 & 1,2 & & $-s m-$ & 1 & 0,1 \\
\hline & $-r(k) k-$ & 21 & 1,1 & & $-s \nu-$ & 1 & 0,1 \\
\hline & $-l v-$ & 18 & 0,9 & & $-t h-$ & 1 & 0,1 \\
\hline & \multirow[t]{2}{*}{$-r(s) s-$} & \multirow[t]{2}{*}{17} & \multirow[t]{2}{*}{0,9} & 46. & $-t m-$ & 1 & 0,1 \\
\hline & & & & Insg & & 1931 & 00,0 \\
\hline
\end{tabular}


Tabelle 12b: Die absolute und prozentuale Frequenz der an der Grenze zwischen erster und zweiter Silbe auftretenden Konsonantenverbindungen im KMS

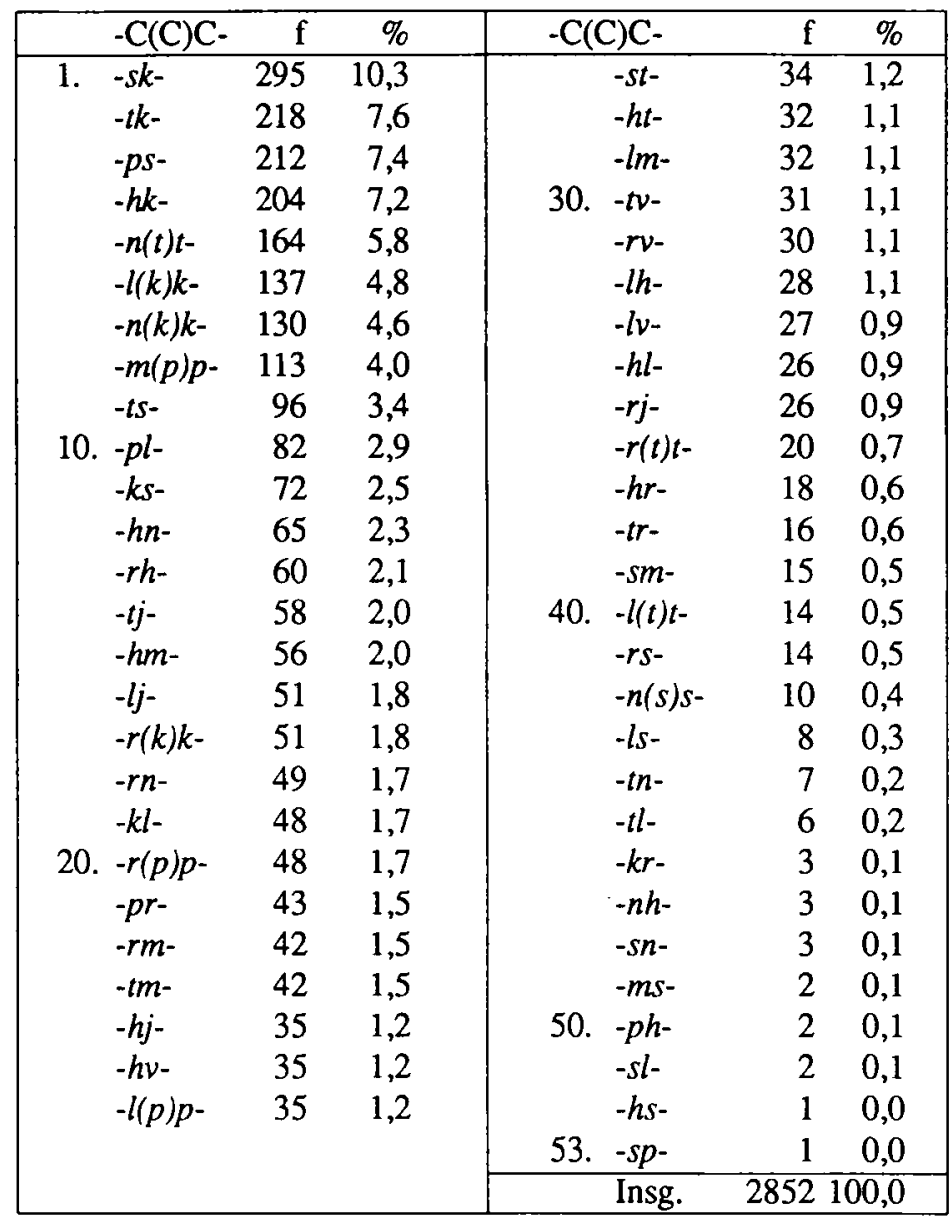

Der Unterschied zwischen dem Wörterbuch der Schriftsprache und dem Dialektwörterbuch tritt erneut deutlich hervor, obwohl die Spitzenpositionen auf der Frequenzliste im großen und ganzen übereinstimmen. Auffällig ist vor allem, daß das Material des KMS 
wesentlich umfangreicher und vielseitiger ist als das des NS: Die Gesamtzahl der Belege beträgt im ersteren 2852, im letzteren nur 1931, und das KMS verzeichnet 53 verschiedene Konsonantenverbindungen, das NS 46. Zwar erscheinen die Verbindungen -skund -ps- im NS deutlich häufiger als im KMS, doch in den meisten anderen Fällen ist das Verhältnis erwartungsgemäß umgekehrt. Beim Material des KMS ist besonders die Häufigkeit der Verbindung $t s$ sowie der Kombinationen Klusil + Liquida, Klusil + Nasal und Klusil + Halbvokal zu beachten (z. B. $-t s-96,-k l-48,-p l-82,-p r-43$, $-t m-42,-t j-58$ und $-t v-31$ ). Der Mundart von Kiihtelysvaara wie generell den heutigen Ostdialekten des Finnischen ist die Verbindung -ts- nämlich fremd, da sie sich regelmäßig zu - $h t$ - entwickelt hat; die Verbindung $t s$ begegnet also nur in den Expressiva. Verbindungen von Klusil und Liquida, Nasal oder Halbvokal wiederum sind seit jeher typisch für die finnischen Ostdialekte, während sie in den wichtigsten Westdialekten, also im SüdwestDialekt und im Dialekt von Häme, selbst in Expressiva nur selten verwendet wurden. Andererseits reicht ihr Verbreitungsgebiet ohne Einschnitte von den Ostdialekten des Finnischen bis ins Karelische und teils auch in die anderen östlichen ostseefinnischen Sprachen.

Tabelle 13 ist eine Zusammenstellung von Angaben über die Verwendung der oben erwähnten Konsonantenverbindungen in den nördlichen osfi. Sprachen, für die mehrere Quellen ausgewertet wurden (Suomen murteiden sanakirja I-II [Finnisches Dialektwörterbuch I-II] und Suomen murteiden sanakirjan arkisto [Archiv des finnischen Dialektwörterbuchs]; Karjalan kielen sanakirja I-III [Wörterbuch des Karelischen I-III] und Karjalan kielen sanakirjan arkisto [Archiv des Wörterbuchs des Karelischen]; Kujola, Lyydiläismurteiden sanakirja [Wörterbuch der lüdischen Dialekte]; Zajceva Mullonen, Словарь вепсского языка [Wörterbuch des Wepsischen]; Nirvi, Inkeroismurteiden sanakirja [Wörterbuch der ingrischen Dialekte]). Die Ziffern geben die Anzahl der Wortstämme an, in denen die einzelnen Konsonantenverbindungen in dem Material aus der jeweiligen Sprache auftreten. 
Tabelle 13: Das Auftreten von zehn inlautenden Konsonantenverbindungen in der expressiven Lexik der nördlichen osfi. Sprachen (fiW. = Westdialekte des Finnischen, fiO. = Ostdialekte des Finnischen, kar. = Karelisch, lüd. = Lüdisch, weps. $=$ Wepsisch, ingr. = Ingrisch)

\begin{tabular}{|l|r|r|r|r|r|r|}
\hline (C)VCC- & fiw. & fiO. & kar. & lüd. & weps. & ingr. \\
\hline (C)Vtsa- & 7 & 78 & 60 & 39 & 31 & 9 \\
(C)Vkl- & 2 & 52 & 44 & 4 & 5 & 4 \\
(C)Vkr- & 0 & 21 & 13 & 0 & 0 & 1 \\
(C)Vpl- & 8 & 58 & 44 & 3 & 4 & 8 \\
(C)Vpr- & 11 & 47 & 41 & 4 & 2 & 4 \\
(C)Vtj- & 4 & 52 & 19 & 1 & 0 & 1 \\
(C)Vtl- & 3 & 20 & 3 & 0 & 0 & 0 \\
(C)Vtm- & 0 & 33 & 19 & 1 & 0 & 1 \\
(C)Vtr- & 1 & 62 & 27 & 1 & 0 & 4 \\
(C)Vtv- & 2 & 34 & 34 & 3 & 1 & 1 \\
\hline Insg. & 38 & 457 & 304 & 56 & 43 & 33 \\
\hline
\end{tabular}

Tabelle 13 veranschaulicht vor allem die enge Zusammengehörigkeit zwischen den finnischen Ostdialekten und dem Karelischen. Die Anzahl der expressiven Wortstämme, in denen zwischen der ersten und der zweiten Silbe eine der in der Tabelle aufgeführten Konsonantenverbindungen auftritt, ist in diesen beiden Sprachformen am größten. Die Differenz gegenüber den anderen Sprachen und Dialekten ist so deutlich, daß sie nicht ausschließlich auf den unterschiedlichen Umfang der Korpora zurückgeführt werden kann. Demnach scheint es sich nur bei der Verwendung der Verbindung (oder Affrikate) $t s$ in Expressiva um ein gemeinsames Charakteristikum aller östlichen osfi. Sprachen zu handeln. Verbindungen von Klusil und Liquida, Nasal oder Halbvokal sind dagegen nur für die finnischen Ostdialekte und für das Karelische besonders charakteristisch.

5. Über die Häufung der Mittel zum Ausdruck der Expressivität

Bei der phonologisch-phonotaktischen Betrachtung des Materials wurde bereits festgestellt, daß bestimmte Laute und Lautverbindungen 
in der expressiven Lexik außerordentlich häufig verwendet werden. Überdurchschnittlich häufig auftretende Konsonanten sind u. a. $h, r$ und $l$, deren absolute Anzahl ebenfalls so hoch ist, daß sie die Frequenzliste der Konsonanten anführen. So bilden etwa im NS die auf $h$ - und auf $r$ anlautenden Expressiva (jeweils 672) die zweitgrößte, die auf $l$ - anlautenden (607) die drittgrößte Gruppe; nur $k$ - ist als anlautender Konsonant häufiger (958). Die bevorzugten Vokale in der expressiven Lexik sind $\ddot{o}, \ddot{a}$ und $y, \mathrm{~d} . \mathrm{h}$. die im übrigen Wortschatz am wenigsten gebräuchlichen Vokale.

Wie Tabelle 13 zeigt, werden in den finnischen Ostdialekten bedeutend häufiger und vielseitiger als in den Westdialekten inlautende Konsonantenverbindungen zum Ausdruck der Expressivität eingesetzt. Andererseits begegnen in den Westdialekten Ausdrucksmittel für die Expressivität, die in den Ostdialekten ganz unbekannt sind. Die wichtigsten sind der anlautende stimmlose Labiodental $f$ sowie die anlautenden Konsonantenverbindungen $f l, f r, k l, k n, k r, t r, p l$ und $p r, z$, B. färistä fyristä 'summen, schnurren', färähtää 'aufschrillen, knacken'; flitistä ja flätistä 'plitschen und platschen', floiskahtaa 'plätschern, überschwappen'; fronkkia 'stochem, drauflosstechen', frutkuttaa 'stampfen'; klapsahtaa 'klappern', klepsua 'schwanken, wanken', klopista 'poltern, klopfen'; knaputtaa 'pochen, klopfen'; krapista 'rascheln', krohista 'röcheln, hüsteln', kränistä 'weinen, jammern'; triputella 'tröpfeln'; plitistä 'plitschen, platschen', pläiskiä 'klatschen, schlagen', pläjähtää 'klatschen, knallen'; pritistä ja prätistä 'knistern und knastern', präiskyä 'krachen, zischen'. Solche Wortformen begegnen in Dialektwörterbüchern; in anderen Wörterbüchern sind sie dagegen eine Seltenheit, da sie nicht der finnischen Schrift- und Hochsprache zugeordnet werden. Bekanntlich sind sowohl anlautendes $f$ als auch Konsonantenverbindungen im Anlaut relativ spät im Finnischen heimisch geworden: Sie wurden erst im ausgehenden Mittelalter zusammen mit schwedischen Lehnwörtern von den süd- und westfinnischen Küstendialekten übernommen (s. z. B. Rapola 1966: 18-19). Ursprünglich nur in Lehnwörtern gebräuchlich, griffen sie später auf expressive Ausdrücke und einige andere Erbwörter über.

In den östlichen verwandten Sprachen des Finnischen, d. h. im Karelisch-Olonetzischen, Lüdischen, Wepsischen und Ingrischen, werden 
fast alle phonologischen Mittel zum Ausdruck und zur Intensivierung der Expressivität verwendet, die auch für die verschiedenen Sprachformen des Finnischen typisch sind. Darüber hinaus begegnen in diesen Sprachen zahlreiche aus dem Russischen entlehnte expressive Merkmale, die im Finnischen weitgehend unbekannt sind. Besonders auffällig sind die folgenden Erscheinungen.

1) Anlautende stimmhafte Klusile, vor allem $b$-. Einige Beispiele aus den einzelnen Sprachen: karS. und ol. boris/sa, -ta 'brodeln; sieden', bärineh 'Geratter, Gerassel', dissa 'Zitze, weibliche Brust', gurbištuo 'sich krümmen, sich beugen' (< russ. gorbit'); lüd. bul'skahtada '(im Wasser) planschen', böristä 'schnurren, summen, brummen', dudišta 'bibbern, zittern', gomahtazetta 'poltern, krachen'; weps. bulahtada '(ins Wasser) plumpsen', bölbotada 'plappern, undeutlich sprechen', dudād'ab '(er) bibbert, zittert', gurbištuda 'sich beugen'; ingr. Laukaansuu bamahtā 'knallen, krachen'.

2) Anlautende Konsonantenverbindungen, z. B. kar.-ol. blakuttoa 'klopfen', bločku 'Knall, Klatschen', gluglattua vom Schwan: 'singen', grabineh 'Geraschel', griž́istä 'knistern, knastern', klokettoa 'klopfen', kröhistä 'röcheln, husten', pläč́kätä 'knallen, klatschen', prötkütteä 'sich schlaff bewegen', stankuttoa 'zanken, keifen', truiskahtoa 'hervorspritzen, auffliegen'; lüd. bringuttada 'spritzen', brötkädä '(z. B. im Sumpf) waten', klikkida 'hinken, humpeln', kraž́aida 'knastern, knistern', plakahtada 'krachen', präč́kähtädä 'knattern, prasseln', šlipettädä 'labbern, schnell schöpfen', šmač́kuttada 'schmatzen'; weps. brägetada vom Schaf: 'mähen', brängutada 'klappern', brökötada 'wiehern', klukkuda vom Huhn: 'gackern', krepsahtada 'mit einem dumpfen Ton fallen', prakutada 'klopfen, pochen', pröngitada '(etwas) kraftvoll, schwungvoll tun', šlängähtada 'plumpsen, klatschen', šmätkähtada 'dumpf fallen', strangahtada 'hinfallen, auffliegen'; ingr. klaputtā '(in die Hände) klatschen', kribissä 'knarren', plajahuttā 'klatschen, knallen', prägissä 'knastem', šlimada 'knallen', šnibristä 'schmerzen, brennen', trilissä 'klingeln, klirren'.

3) Anlautendes $f$ (meist nur in russischen Lehnwörtern), z. B. karS. und ol. firahuttua '(etwas) schnell tun, sausen', lüd. furišta 'rascheln; summen', weps. fuzaita 'säuseln, zischen', ingr. fursk $\bar{a}$ 'mit dem Mund, der Nase oder den Nüstern schnauben'. 


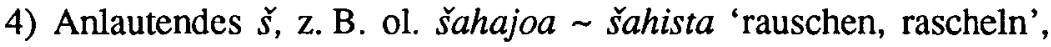
šolišta 'rieseln', šuhettoa 'säuseln', šupettoa 'tuscheln'; lüd. heińäd šahistau 'das Heu raschelt', šonaitta 'rieseln', šuhettada 'flüstern'; weps. šihaita 'zischen', šobaita šobišta 'rascheln', šunaita 'klingeln'; ingr. Laukansuu, Rosona šahissa 'rascheln', šikkärä 'Locke, lockig', šlkkia 'stochern, umrühren', šukutella 'tuscheln'.

5) Anlautende und inlautende Affrikate, z. B. kar.-ol. ćahista 'zischen, pfeifen', čič́etteä 'kichern müssen, Niesreiz verspüren', č́okerdoa 'an etwas herumbasteln', ćuglahtoa 'hervorquellen', ćäbristüö 'einschrumpfen, sich ringeln', čökkie 'knuffen, stechen', hüč́istüö 'sich kauern, sich krümmen', ri ̌̌issä 'knistern', nǚ̆ ä 'Stummelpfeife', nič́koa 'knirschen, knarren', hä̋̆vä 'Kohlendunst, Rauch', kurč́istuo 'schrumplig werden, zusammenfallen', län žäkkä 'schlammig, schmutzig'; lüd. čiketteä 'zirpen', č orišta 'murmeln; rieseln', ćuhišta 'säuseln, zischen',

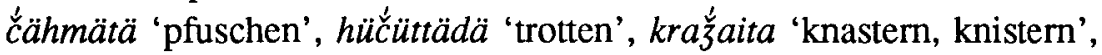
räč́käitä vom Donner: 'krachen', murč́ištuda 'sich biegen, sich krümmen'; weps. čihištä 'zischen', čirkta 'tschilpen', čonkta 'wühlen', čoraita 'brummen, knurren', čuhkta 'ächzen, grollen', čirk 'lebhaft', čiksutada 'zwitschem, ticken', hačatada von der Elster: 'lachen, schwatzen', ńäćotada 'schmatzend essen', kožaita 'knarren', vǐ̌́eita 'piepsen', лoč́keita лoč́kęita 'dumpf tönen lassen', virčištada 'grinsen'; ingr. (Affrikate nur im Anlaut) čahissa 'rascheln', č́arahtā 'auflodern',

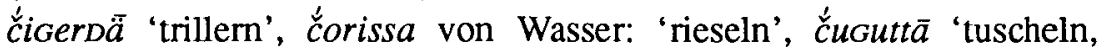
zischeln', čärissä 'rasseln, knastern'.

6) Anlautendes palatalisiertes $n$, z. B. kar.-ol. ńaksuttoa 'schnalzen', ńaukuo ńauguo 'wimmern, miauen', ńorskuo 'knirschen', ñuhissa 'näseln'; lüd. ñapsahtada 'knacken', naukkida 'nach etwas schnappen', norahtazetta 'ausfließen'; weps. ńaukta 'nach etwas schnappen', nola 'klatschnaß', ńouskahtada 'mit einem dumpfen Ton fallen', ńärista 'wimmern, klagen'.

7) Inkonsequent palatalisierte dentale Konsonanten, vor allem -l'-, in anlautenden Konsonantenverbindungen und inlautend zwischen Hintervokalen, z. B. kar.-ol. kl'a $k$ koa 'plätschern, schwappen; faulenzen',

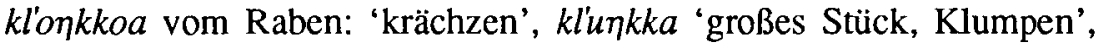
mul'ahtoakseh 'plötzlich etwas sagen, aufbrüllen', mul'ata : mul'koau 'finster anblicken', smul'ahuttoa 'finster anblicken, anglotzen', hońnot- 
toa 'zu tun vorgeben', bal'skuttoa 'verschütten', mul'mettoa 'undeutlich sprechen', bat'ma 'Gerte, Rute', kutkuttoa vom Huhn: 'gackeln', kut'makko 'ärmlich gekleidet; still, langsam'; lüd. mul'u '(Kuh) ohne Hörner', uśśsttada uśuttada '(den Hund auf einen) hetzen', bal'bettada 'reden, plappern', bul'skahtada 'plumpsen, platschen', kud'vahtada 'sich bewegen', kut'kutez 'Juckreiz, Kitzeln'; weps. bul'ahtada '(ins Wasser) plumpsen', núña 'Penis (eines kleinen Jungen)', huśutada uśutada '(den Hund auf einen) hetzen', mull' : mul'lun 'Beule', bul'botada vom Wasser: 'glucksen', bul'skahus 'Plumpsen', pal'patada 'plappern', huńhutada 'näseln', kứñăda 'wimmern, miauen', kut'kutada 'kitzeln'.

8) In der übrigen Lexik unbekannte inlautende Konsonantenverbindungen, vor allem -kn- und -pn-; z. B. kar.-ol. fukńie $\sim$ hukńie 'sich schnell oder kraftvoll bewegen' (< russ. fuknut'), hakñie 'schnell oder hastig essen', hikńie 'laut sprechen', sokńie vom Regen: 'nieseln', sukńie 'heftig schlagen', takńie 'scharf widersprechen', č́okñie '(etwas) plötzlich tun; schlagen', äkñie 'schnell oder heftig stechen', hapñie 'hastig essen, nach etwas schnappen', hipnie 'sich schnell bewegen', sapnie 'fest schlagen', sipñie 'streifen, flüchtig berühren', tapnie täpñie 'heftig zerren'; lüd. fukńida 'sich schnell bewegen, dahinrasen', heikńidä 'hallo rufen', hüpńidä 'aufspringen, auffahren'; weps. fukńida 'sich schnell bewegen, dahinrasen', žogñida '(etwas) plötzlich tun; schlagen', hлopnida 'klatschen, knallen' (< russ. hlopnut'), hüpńida 'aufspringen'.

Durch das Auftreten der Verbindungen - $k n$ - und -pn-verändert sich die Phonotaktik der expressiven Lexik in den östlichen osfi. Sprachen radikal. Zwar kannten diese Sprachen seit jeher mehrere Verbindungen von Klusil + Liquida und Klusil + Nasal $(k l, k r, t l, t m$, $t n, t r, p l, p r)$, doch nun ist die Reihe nahezu lückenlos; es fehlen nur ${ }^{*} \mathrm{~km}$ und $* p m$. Dies wird durch die folgenden Diagramme veranschaulicht, die die entsprechenden Vertretungen in den Ostdialekten des Finnischen und im Karelischen zeigen.

Ostdialekte des Finnischen

\begin{tabular}{|l|l|llll|}
\hline $\mathrm{C} 1$ & $\mathrm{C} 2$ & $\mathrm{l}$ & $\mathrm{r}$ & $\mathrm{m}$ & $\mathrm{n}$ \\
$\mathrm{k}$ & & + & + & - & - \\
$\mathrm{t}$ & & + & + & + & + \\
$\mathrm{p}$ & & + & + & - & - \\
\hline
\end{tabular}

Karelisch-Olonetzisch

\begin{tabular}{|l|l|llll|}
\hline $\mathrm{C} 1$ & $\mathrm{C} 2$ & $\mathrm{l}$ & $\mathrm{r}$ & $\mathrm{m}$ & $\mathrm{n}$ \\
$\mathrm{k}$ & & + & + & - & + \\
$\mathrm{t}$ & & + & + & + & + \\
$\mathrm{p}$ & & + & + & - & + \\
\hline
\end{tabular}


In der finnischen Hochsprache sind Verbindungen von Klusil und Liquida fast marginal und Verbindungen von Klusil und Nasal völlig unbekannt (Karlsson 1982: 117, 119). Die Phonotaktik der expressiven Lexik ist in dieser Hinsicht ganz anders geartet: In den Ostdialekten des Finnischen sind nur die Verbindungen $k+$ Nasal und $p+$ Nasal ausgeschlossen, im Karelisch-Olonetzischen nur die Verbindungen $\mathrm{km}$ und $p m$. Die oben erwähnten, durch Lehnwörter vermittelten phonologischen Mittel zum Ausdruck und zur Intensivierung der Expressivität werden in der folgenden Merkmalmatrix dargestellt, bei der die Westdialekte (fiW.) und Ostdialekte (fiO.) des Finnischen, die nordkarelischen oder weißmeerkarelischen Dialekte (karN.), die Süddialekte des Karelischen (karS.) und die olonetzischen Dialekte des Karelischen (ol.) sowie das Lüdische (lüd.), das Wepsische (weps.) und das Ingrische (ingr.) einbezogen wurden.

\begin{tabular}{|l|llllllll|}
\hline Merkmal & fiW. & fiO. & karN. & karS. & ol. & lüd. & weps. ingr. \\
\hline$b-, d-, g-$ & - & - & - & + & + & + & + & -1 \\
$C C-$ & + & - & + & + & + & + & + & + \\
$f-$ & + & - & - & + & + & + & + & + \\
$\check{\xi}-$ & - & - & - & - & + & + & + & -1 \\
$\check{C}-$ u.a.. & - & - & + & + & + & + & + & + \\
$n_{-}-$ & - & - & + & + & + & + & + & - \\
$-l^{\prime},-, t^{\prime},-,-n-$ & - & - & + & + & + & + & + & - \\
$-k n-,-p n-$ & - & - & + & + & + & + & + & - \\
\hline
\end{tabular}

Die Matrix zeigt vor allem, in welch großem Umfang fremdartige lautliche Merkmale aus einer anderen Sprache zum Ausdruck der Expressivität eingesetzt werden können. Auffällig ist ferner der starke Einfluß des Russischen auf die deskriptiv-onomatopoetische Lexik der östlichen osfi. Sprachen. Die Zusammengehörigkeit des Karelischen, Lüdischen und Wepsischen wurde dadurch verstärkt, zugleich vertiefte sich jedoch die Kluft zwischen diesen Sprachen und dem Finnischen. Dies gilt besonders für die finnischen Ostdialekte und das Karelische, deren Expressiva ursprünglich offenbar eine gleichartige phonologische Struktur aufwiesen (s. z.B. Tabelle 12). 


\section{Zu den Bedeutungsrelationen}

Ein Charakteristikum der Expressiva ist bekanntlich ihre semantische Unbestimmtheit; ein abbildender, beschreibender Ausdruck kann wohl gar nicht präzis und exakt definiert sein. Dieses Merkmal der Expressiva stellt - wie oben bereits angemerkt wurde - den Lexikographen immer wieder vor Schwierigkeiten: Er sieht sich häufig gezwungen, die Definition der Bedeutung durch eine Erläuterung zur Verwendung des Wortes oder durch ein bloßes Beispiel zu ersetzen. Dennoch dürfte es, nachdem ich oben die enge formale Zusammengehörigkeit der expressiven Lexik der finnischen Ostdialekte und des Karelischen hervorgehoben habe, angebracht sein, das Material aus diesen beiden Sprachformen zu einem gewissen Grad auch unter inhaltlichem Aspekt zu betrachten. Im folgenden vergleiche ich vier den finnischen Ostdialekten und dem Karelischen gemeinsame expressive Wortstrukturen im Hinblick auf Bedeutung und Verwendungskontext.

1) Auf hats $(V)$ - hać $(V)$ - anlautende Wörter. Diese Wörter beschreiben a) die Stimme der Elster (z. B. fi. V. hatsahtaa, hatsattaa von einer Elster: 'lachen, krächzen', kar. Interj. hač, V. hac̆attoa, S. hač́akka 'Elster'), b) wortreiches, schnelles Sprechen, Streit oder Gezänk (z. B. fi. V. hatsattaa, hatsauttaa, kar. V. hačartoa, haćattoa), c) menschliche Geschäftigkeit, Betriebsamkeit oder Toberei (z. B. fi. S. hatsakka 'geschäftiger Mensch, Betriebsnudel', V. hatsattaa, hatsertaa, hatsitella, hatsottaa 'hastig (etwas) tun', kar. V. hač́artoa id.), d) offenes oder zerzaustes Haar (z. B. fi. V. hatsattaa, olla hatsallaan von den Haaren: 'offen sein, (vom Kopf) abstehen'; kar. keine Belege), e) verdorbene oder verschrumpelte Hackfrüchte, abgestandene Getränke, verblichenen Stoff u.ä. (z. B. fi. V. hatsettua, kar. V. hač́istuo), f) das Aufscheuchen von Tieren (z. B. fi. Interj. hats, kar. V. hač́istoa 'aufscheuchen'), g) Getreidekeime (kar. S. hac̆́akka, -ine; fi. keine Belege). - Die Verwendungsbereiche stimmen weitgehend überein; die Unterschiede könnten als dialektal bezeichnet werden.

2) Auf $l V k l V$ - anlautende Wörter. Charakteristisch für diese Wörter sind in beiden Sprachen die zahlreichen Variationen des inlautenden Vokals. Beispiele: V. laklatt/aa, -oa fi. a) lautes oder wirres Sprechen, b) das Geräusch des sich drehenden Windrads, kar. a) lautes Sprechen, 
b) Vogelstimmen; V. leklett/ää, -eä fi. a) lautes Sprechen, b) das Ticken der Uhr, kar. a) lebhaftes Sprechen, b) das Trinken bei Tieren: 'labbern'; liklett/ää, -eä fi. eine flatternde, wiederholte Bewegung, kar. a) von fließendem Wasser: 'murmeln, rieseln', b) ein gleichmäßig brennendes Feuer, c) eine leichte oder einfache Tätigkeit; V. liklittää fi. ein regelmäßig wiederkehrendes, leises Geräusch: 'plätschern', b) das Trinken bei Tieren: 'labbern', kar. keine Belege; V. loklott/aa, -oa fi. a) unnützes Reden, b) das Geräusch von fließendem Wasser, c) eine wiederkehrende Bewegung, kar. a) lautes oder unnützes Reden, b) das Geräusch von fließendem Wasser: 'murmeln', c) hastiges Essen: 'schlingen'; V. luklettoa kar. a) Vogelstimmen, b) Lallen, c) eine leichte, mühelose Tätigkeit (fi. keine Belege); V. luklottaa fi. a) Vogelstimmen, b) eine leichte Tätigkeit (kar. keine Belege); V. lukluttaa fi. das Quaken der Frösche (kar. keine Belege); V. läklätt/ää, -eä fi. a) nachlässiges oder undeutliches Sprechen, b) eine wiederkehrende oder pulsierende Bewegung: 'flattern, pochen', kar. a) unnützes Reden, b) das Trinken bei Tieren: 'labbern'; V. löklentää fi. undeutliches Sprechen (kar. keine Belege); V. löklötteä kar. a) das Geräusch von fließendem Wasser: 'murmeln', b) Schmatzen beim Essen (fi. keine Belege). Wieder liegen Bedeutung und Verwendungsbereiche eng beieinander.

3) Auf $j V h l V$ - anlautende Wörter. Dieser Worttyp ist im Karelischen recht verbreitet, im Finnischen dagegen selten; z. B. kar. V. jahlie 'etwas wiederkäuen, unaufhörlich wiederholen', S. jahlikka als Bezeichnung für ein dickes und tolpatschiges Kind, V. juhlie a) 'lärmen', b) 'verprügeln', S. juhlu 'steiler Abhang', S. jyhleikkö für einen Wald mit robusten Stämmen, V. jyhlistyö a) vom Gelände: 'hügelig werden', b) vom Himmel: 'sich bewölken, sich verdüstern', S. jyhlö 'steiler Abhang', b) 'dunkle Wolke', c) 'Schwiele, Knoten', Adj. jählie 'uneben, holperig', Adj. jähläkkö id.; im Finnischen begegnen nur die Verben jöhlätä 'langsam und vorsichtig gehen' und jöhlötellä 'untätig sein, faulenzen'. Der Unterschied ist also erheblich, betrifft jedoch eine Wortgruppe mit sehr niedriger Frequenz.

4) Auf $j V h r V$ - anlautende Wörter. Hier ist die Situation genau umgekehrt: Der Worttyp begegnet im Finnischen, ist dagegen im Karelischen unbekannt. Z.B. fi. S. jahrus als Bezeichnung für einen sich langsam bewegenden und tolpatschigen Menschen, V. jahruta 'sich steif bewe- 
gen', V. juhrata '(etwas) langsam tun', S. juhru für einen langsamen und tolpatschigen Menschen, V. jyhrytä '(etwas) langsam tun'. Offensichtlich sind die Typen $j V h l V$ - und $j V h r V$ - verhältnismäßig spät entstanden und haben sich in den beiden Sprachen selbständig entwickelt.

Die Übereinstimmung der Bedeutung und die Gleichartigkeit des Verwendungsbereichs der einzelnen Expressiva in den finnischen Ostdialekten und im Karelischen scheinen so evident, daß die geringfügigen Abweichungen die Auffassung von einer genetischen Zusammengehörigkeit der Grundelemente nicht erschüttern. Diese Auffassung wird auch durch die Angaben der etymologischen Wörterbücher unterstützt: Die dem Finnischen und Karelischen gemeinsamen deskriptivonomatopoetischen Wörter beschreiben in der Regel in beiden Sprachen genau das gleiche. Dagegen bestehen zwischen dem Finnischen und dem Wepsischen sowie zwischen dem Finnischen und dem Estnischen in manchen Fällen erhebliche Bedeutungsunterschiede, die von einer seit langem anhaltenden divergierenden Entwicklung zeugen.

\section{Zum AbschluB}

Das hier vorgestellte Material zeigt deutlich, daß die expressive Lexik ein eigenes, koexistentes System bildet, das sich sowohl im Phonembestand als auch in der Phonotaktik vom normalen System der Sprache unterscheidet. Vor allem ist $\mathrm{zu}$ beobachten, daß in der expressiven Lexik generell Phoneme und Phonemkombinationen auftreten, die im übrigen sprachlichen Material selten oder sogar unbekannt sind. Zudem wurde die expressive Lexik offenbar nicht einmal von allen regelmäßigen Lautveränderungen erfaßt. Ein bezeichnendes Beispiel ist die Entwicklung der Verbindung $t s$ in den Ostdialekten des Finnischen: In einem weiten Gebiet hat sich $t s$ zu ht gewandelt (mehtä 'metsä (Wald)', vihta 'vitsa (Gerte)'), in den Expressiva ist es dagegen erhalten geblieben und offenbar auch produktiv geworden. Die gleiche Beobachtung ist im Fall der Verbindungen von Klusil und Liquida zu machen: Das Wort kakla 'Hals' hat wahrscheinlich schon vor einigen Jahrhunderten in den meisten östlichen Dialekten des Finnischen die Form kaula, das 
Wort kakra 'Hafer' die Form kaura angenommen, usw.; dennoch blieben in der expressiven Lexik die Verbindungen $k l$ und $k r$ erhalten. Man könnte sogar sagen, daß sich der expressive Effekt der Verbindungen noch verstärkt hat, da sie in der normalen Sprache nicht mehr verwendet werden. Entsprechend wurden die Expressiva in den Weißmeerund Süddialekten des Karelisch-Olonetzischen nicht von dem relativ alten Stufenwechsel $t k$ : $t$ erfaßt: Zwar hat die Flexion kätkyön (Gen. Sg.) : kätyt (Nom. Sg.) 'Wiege', matka (Nom. Sg.) : matalla (Adess. Sg.) 'Reise', pitkä (Nom. Sg.) : pitän (Gen. Sg.) 'lang' ausschließliche Geltung, doch in den Expressiva ist abweichend von dieser Regel die Verbindung $t k$ auch in geschlossener Silbe starkstufig, z. B. hytkytteä 'schaukeln, wiegen', mutkahtoa 'dumpf hinfallen' usw. Gerade die Unveränderlichkeit der Verbindung $t k$, d.h. die Abweichung von den Regeln der normalen Sprache, ist also zum Kennzeichen der Expressivität geworden.

Ein zweiter wichtiger Umstand, der angesichts des Materials aus den östlichen ostseefinnischen Sprachen berücksichtigt werden muß, ist der starke Einfluß einer fremden Sprache auf die Entwicklung der Expressiva. Dies zeigt sich bereits in den finnischen Dialekten, tritt jedoch im Karelisch-Olonetzischen, Lüdischen und Wepsischen besonders deutlich zutage. Die expressive Lexik ist zweifellos der Weg, auf dem neue Lautmerkmale in das alte Lautsystem vordringen können, sofern die Sprecher sie zuvor durch direkte Entlehnungen kennengelernt haben. Dies ist an sich nichts Neues, doch dürfte es angebracht sein, darauf hinzuweisen, daß bei einem reichen Angebot an affektiv gefärbten oder als affektiv aufgefaßten Elementen fremder Herkunft eigenständige Ausdrucksmittel verdrängt oder an ihrer vollen Entfaltung gehindert werden. Dies scheint zumindest im Lüdischen und Wepsischen geschehen zu sein. Eine parallele Entwicklung läßt sich auch in den Westdialekten des Finnischen aufzeigen, die bekanntlich stark vom Schwedischen beeinflußt wurden.

Die geographische Verbreitung der hier behandelten Typen expressiver Strukturen erlaubt einige vorsichtige Schlußfolgerungen, deren Zuverlässigkeit allerdings durch die Lückenhaftigkeit des lüdischen, wepsischen und ingrischen Materials beeinträchtigt wird. Vor allem bietet die expressive Lexik ein schlagkräftiges Argument für eine enge 
Zusammengehörigkeit der nördlichen ostseefinnischen Sprachen, also des Finnischen, Karelisch-Olonetzischen, Lüdischen, Wepsischen und Ingrischen. Besonders nahe scheinen sich die Ostdialekte des Finnischen und das Karelisch-Olonetzische zu stehen: Beide Sprachformen nutzen in geradezu verblüffendem Ausmaß die gleichen lautlichen Mittel zum Ausdruck der Expressivität. Sowohl in westlicher wie in östlicher Richtung ist der Unterschied so schroff, daß man auch für die expressive Lexik dieser Sprachen eine gemeinsame altkarelische Herkunft annehmen muß. Andererseits sind auch deutliche Hinweise - vor allem die Vorliebe für die Verbindung $t s$ - auf enge Beziehungen zwischen allen östlichen ostseefinnischen Sprachen zutage getreten. Allerdings haben das Wepsische, Lüdische und Karelisch-Olonetzische sowie teils auch das Ingrische infolge des anhaltenden und kontinuierlich zunehmenden russischen Einflusses expressive Merkmale übernommen, die in den Ostdialekten des Finnischen unbekannt sind. Die expressive Lexik scheint infolgedessen nicht nur von der Vergangenheit und Gegenwart der Sprache Zeugnis abzulegen, sondern auch wertvolle Hinweise auf ihre Zukunft zu geben.

\section{Literatur}

HakUlinen, Lauri 1979: Suomen kielen rakenne ja kehitys (Struktur und Entwicklung der finnischen Sprache). Vierte, revidierte und erweiterte Auflage. Keuruu.

ITKONEN, ERKKI 1966: Kieli ja sen tutkimus (Sprache und Sprachforschung). Helsinki.

KARLSSON, FRED 1962: Suomen kielen äänne- ja muotorakenne (Die Laut- und Formstruktur der finnischen Sprache). Juva 1983.

KKS = Karjalan kielen sanakirja I-III (Wörterbuch des Karelischen I-III). Chefredakteur PertTi VIRTaranta. Lexica Societatis Fenno-Ugricae XVI: 1-3. Helsinki 1968, 1974, 1983.

KMS = R. E. NIRVI, Kiihtelysvaaran murteen sanakirja I-X (Wörterbuch der Mundart von Kiihtelysvaara I-X). Joensuu 1974-1981. 
Kujola, JuHo 1944: Lyydiläismurteiden sanakirja (Wörterbuch der lüdischen Dialekte). Lexica Societatis Fenno-Ugricae IX. Helsinki.

Makarov, G. N. 1990 = МАКАРОв, Г. Н.: Словарь карельского языка (Wörterbuch des Karelischen). Петрозаводск.

NIRVI, R. E. 1971: Inkeroismurteiden sanakirja (Wörterbuch der ingrischen Dialekte). Lexica Societatis Fenno-Ugricae XVIII. Helsinki.

NS = Nykysuomen sanakirja 1-6 (Wörterbuch der finnischen Gegenwartssprache 1-6). Porvoo 1951-1961.

OJANSUU, HEIKKI 1918: Karjala-aunuksen äännehistoria (Lautgeschichte des Karelisch-Olonetzischen). Suomalaisen Kirjallisuuden Seuran toimituksia 162. Helsinki.

RAPOLA, MARTTI 1966: Suomen kielen äännehistorian luennot (Vorlesungen zur Lautgeschichte des Finnischen). Suomalaisen Kirjallisuuden Seuran toimituksia 283. Helsinki.

RaVILA, PaAvo 1967: Onomatopoeettisten ja deskriptiivisten sanojen asema kielen äännesysteemissä (Die Stellung der onomatopoetischen und deskriptiven Wörter im Lautsystem der Sprache). - Totuus ja metodi. Porvoo.

RYTKÖNEN, AHTI 1940: Eräiden itämerensuomen tm-sanojen historiaa (Zur Geschichte einiger ostseefinnischer $t m$-Wörter). Jyväskylä.

SivUla, JAAKKo 1989: Deskriptiiviset sanat (Die Deskriptiva). - Nykysuomen sanavarat. Hg. von JouKo VESIKANSA. Juva.

SKES = Suomen kielen etymologinen sanakirja I-VI (Etymologisches Wörterbuch des Finnischen I-VI). Hg. von Y. H. TOIVONEN, ERKKI Itkonen, Aulis J. Joki und Reino Peltola. Lexica Societatis FennoUgricae XII. Helsinki 1955-1978.

SMS = Suomen murteiden sanakirja 1-2 (Finnisches Dialektwörterbuch 1-2). Chefredakteur Tuомо Tuomi. Kotimaisten kielten tutkimuskeskuksen julkaisuja 36. Helsinki 1985, 1988.

SSA = Suomen sanojen alkuperä. Etymologinen sanakirja 1 A-K (Die Herkunft der Wörter des Finnischen. Etymologisches Wörterbuch 1 A-K). Chefredakteure ERKki ItKonEn und Ulla-Maija Kulonen. Suomalaisen Kirjallisuuden Seuran toimituksia 556, Kotimaisten kielten tutkimuskeskuksen julkaisuja 62. Jyväskylä 1992.

TuомI, TUомо 1989: Yleiskielemme murrepohjainen sanasto (Der mundartliche Wortschatz in der finnischen Hochsprache). - Nykysuomen sanavarat. Hg. von Jouko VesiKansa. Juva.

WIIK, KALEVI 1969: Onko suomen yleiskielessä oppositioita /i/-/j/ ja / / / $-/ \mathrm{v} /$ ? (Gibt es in der finnischen Hochsprache die Oppositionen $/ \mathrm{i} / \mathrm{-} / \mathrm{j} / \mathrm{und} / \mathrm{u} /$ -/v/?). - Juhlakirja Paavo Siron täyttäessä 60 vuotta. Acta Universitatis Tamperensis A: 26. Tampere.

Zajceva, M. - Mullonen, M. 1972 = ЗАйцевА, М. - Муллонен, М.: Словарь вепсского языка (Wörterbuch des Wepsischen). Ленинград. 
Karjalan kielen sanakirjan arkisto. Kotimaisten kielten tutkimuskeskus (Archiv des Wörterbuchs des Karelischen. Forschungszentrum für die Landessprachen Finnlands). Helsinki.

Suomen murteiden sana-arkisto. Kotimaisten kielten tutkimuskeskus (Finnisches Dialektarchiv. Forschungszentrum für die Landessprachen Finnlands). Helsinki. 